\title{
Matroids, Cyclic Flats, and Polyhedra
}

\section{Kadin Prideaux}

A thesis

submitted to the Victoria University of Wellington

in fulfilment of the requirements for the degree of

Master of Science

in Mathematics.

Victoria University of Wellington

2016 



\begin{abstract}
Matroids have a wide variety of distinct, cryptomorphic axiom systems that are capable of defining them. A common feature of these is that they are able to be efficiently tested, certifying whether a given input complies with such an axiom system in polynomial time. Joseph Bonin and Anna de Mier, rediscovering a theorem first proved by Julie Sims, developed an axiom system for matroids in terms of their cyclic flats and the ranks of those cyclic flats. As with other matroid axiom systems, this is able to be tested in polynomial time. Distinct, non-isomorphic matroids may each have the same lattice of cyclic flats, and so matroids cannot be defined solely in terms of their cyclic flats. We do not have a clean characterisation of families of sets that are cyclic flats of matroids. However, it may be possible to tell in polynomial time whether there is any matroid that has a given lattice of subsets as its cyclic flats. We use Bonin and de Mier's cyclic flat axioms to reduce the problem to a linear program, and show that determining whether a given lattice is the lattice of cyclic flats of any matroid corresponds to finding integral points in the solution space of this program, these points representing the possible ranks that may be assigned to the cyclic flats. We distinguish several classes of lattice for which solutions may be efficiently found, based upon the nature of the matrix of coefficients of the linear program, and of the polyhedron it defines, and then identify families of lattice that belong to those classes. We define operations and transformations on lattices of sets by examining matroid operations, and examine how these operations affect membership in the aforementioned classes. We conjecture that it is always possible to determine, in polynomial time, whether a given collection of subsets makes up the lattice of cyclic flats of any matroid.
\end{abstract}




\section{Acknowledgments}

I would like to thank my supervisor, Dillon Mayhew, without whose untiring assistance, this work would not be possible.

Further thanks go to Joe Bonin and Anna de Mier, whose work inspired this. 


\section{Contents}

1 Introduction 1

1.1 Independent sets, bases, and circuits of matroids . . . . . . . . 1

1.2 Ranks, closures, and flats . . . . . . . . . . . . . 2

1.3 Duals, loops, and coloops . . . . . . . . . . . . . . 2

1.4 Visualising matroids . . . . . . . . . . . . . . . 3

1.5 Cryptomorphisms and certification of matroids . . . . . . . 3

1.6 Cyclic flats . . . . . . . . . . . . . . 6

2 Preliminaries 17

2.1 Matroid minors . . . . . . . . . . . . . . . . . 17

2.2 Lattices . . . . . . . . . . . . . . . . . . . 17

2.3 Linear and integer programming . . . . . . . . . . . . 19

2.4 Matrices . . . . . . . . . . . . . . . . . . 22

2.5 Cyclic flat axioms as a system of inequalities . . . . . . . . . 22

2.6 Elementary results concerning cyclic flats . . . . . . . . . . . . 26

3 Special classes of lattices 29

3.1 Loops and coloops . . . . . . . . . . . . . . . . . . . . . 29

3.2 Lattices of height $3 \ldots \ldots \ldots$. . . . . . . . . . . 30

3.3 Certain types of lattice of width at most $2 \ldots . . \ldots 33$

4 Sums and products of lattices $\quad 37$

4.1 Direct sums . . . . . . . . . . . . . . . . . . . . . 37 
4.2 Free products . . . . . . . . . . . . . . . . 40

5 Duals 49

5.1 Lattice duals . . . . . . . . . . . . . . . . . . . . . 49

5.2 Polyhedra of dual lattices . . . . . . . . . . . . . . 51

6 Lattice minors $\quad 57$

6.1 Defining lattice minors . . . . . . . . . . . . . 57

6.2 Minor-closed classes of lattice . . . . . . . . . . . . . . 61

$\begin{array}{ll}\text { Appendices } & 71\end{array}$

$\begin{array}{ll}\text { A Software } & 73\end{array}$ 


\section{Chapter 1}

\section{Introduction}

\subsection{Independent sets, bases, and circuits of matroids}

A matroid is a kind of discrete geometric structure able to be represented in a number of different ways. Many such representations are in the form of a set of elements $E$ called the ground set of a matroid, and a collection of distinguished subsets of $E$. Matroids are commonly described in terms of their independent sets, a collection $\mathcal{I}$ of subsets of the ground set.

Definition 1. A matroid $M$ is a pair $(E, \mathcal{I})$, where $E$ is a finite set and $\mathcal{I} \subseteq 2^{E}$ is the collection of independent sets of $M$, and $\mathcal{I}$ satisfies:

1. $\mathcal{I}$ is nonempty.

2. If $X \in \mathcal{I}$ and $Y \subseteq X$, then $Y \in \mathcal{I}$.

3. If $X, Y \in \mathcal{I}$ and $|X|>|Y|$, then there exists some $x \in X \backslash Y$ such that $Y \cup\{x\} \in \mathcal{I}$.

A basis of a matroid is a maximal independent set, a set $B \subseteq E$ that is independent such that every proper superset of $B$ is dependent. It follows from the independent set axioms that all bases of a matroid $M$ must have the same cardinality. It is easy to see that the independent sets of a matroid 
can be trivially derived from its bases, simply by taking all subsets of those bases.

Another kind of distinguished set in a matroid is a circuit. A circuit of a matroid is a subset of $E$ that is minimally dependent, i.e., a set $C \subseteq E$ such that $C$ is not independent, but every proper subset of $C$ is independent.

\subsection{Ranks, closures, and flats}

A matroid $M$ has a rank function $r$ that takes subsets of $E$ to nonnegative integers. If $X \subseteq E$, then $r(X)$ is simply the size of the largest independent subset contained in $X$. For example, if $X$ is an independent set itself, then $r(X)=|X|$. The rank of the ground set $E$ is the cardinality of a basis $B$ of $M$, and is said to be the rank of the matroid.

The closure of a set $X \subseteq E$ is the largest superset $Y \subseteq E$ of $X$ such that $Y$ has the same rank as $X$. For example, the closure of any basis $B$ clearly must be the ground set $E$.

A flat of a matroid is a subset $F \subseteq E$ such that the closure of $F$ is equal to $F$. The closure of $E$ obviously will be $E$, therefore the ground set $E$ is a flat of $M$.

\subsection{Duals, loops, and coloops}

The dual of a matroid $M$ is a matroid $M^{*}$ that has the same ground set as $M$, but which has as its bases the complements of the bases of $M$, i.e., if $M$ has ground set $E$, and $B$ is a basis of $M$, then $E \backslash B$ is a basis of $M^{*}$.

A loop in a matroid $M$ is an element that has a rank of 0 in $M$. A loop is therefore not contained in any independent set of $M$.

A coloop or isthmus of a matroid $M$ is an element that has a corank of 0 , a rank of 0 in the dual of $M$. If $x$ is a coloop of $M$ and $B$ is a basis of $M$, then $x \in B$. 


\subsection{Visualising matroids}

Matroids have an elegant geometric representation that is able to aid in understanding many concepts in matroid theory.

Given a matroid $M$ with ground set $E$ and rank $r$, the elements of $E$ are distributed in a space of dimension $r-1$ (so a rank-3 matroid is represented by points on a plane, for example), where points may be placed at the same location (usually depicted as separate yet adjacent points), and connected by some lines. In this representation, a set of two points is dependent if they have the same location; a set of three points is dependent if they are colinear; a set of four points is dependent if they are coplanar; and so on. Loops (dependent sets of size one) are represented by points set off to the side and enclosed in a box, separate from all other elements.

It is easy to see many aspects of a matroid by looking at such a representation. For example, in Figure 1.4.1 on page 4, each set of points lying on a line is a flat, as is the set of three points all drawn touching, as well as the set of all points in the diagram. All points are drawn in the same plane, so there is no independent set of size 4 ; it is a rank-3 matroid. There are many visible circuits: any pair of the three points that are touching each other form a circuit, as does any set of three points in a line, provided that no two of them belong to the set of the three adjacent points (that is, provided the set of three points contains no smaller circuit). Dependent sets of points are those that contain circuits.

\subsection{Cryptomorphisms and certification of matroids}

In Section 1.1, matroids were defined in terms of their independent sets. There are many equivalent descriptions of matroids, which are said to be cryptomorphic to one another. There are matroid axioms for bases, circuits, rank functions, closures, and flats.

For example, matroids are characterised in terms of their circuits with 


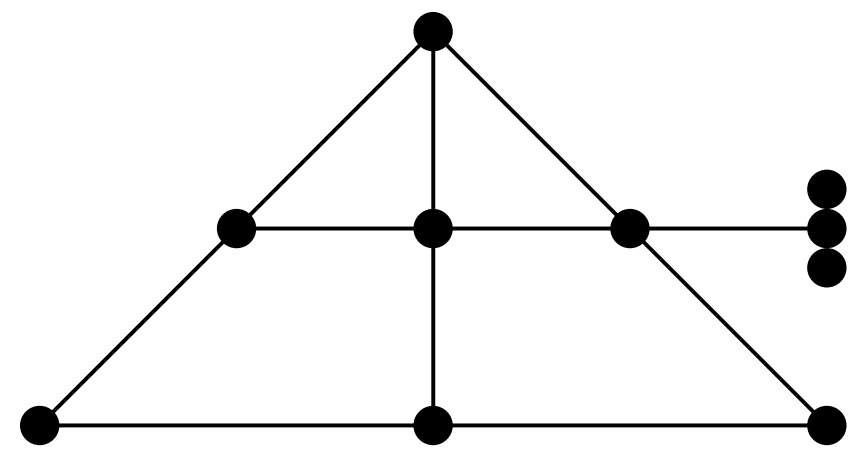

Figure 1.4.1: A matroid

the following axioms.

Theorem 2. Given a finite set $E$ and a collection $\mathcal{C} \subseteq 2^{E}$ of subsets of $E$, there exists a matroid $M$ on $E$ that has $\mathcal{C}$ as its collection of circuits if and only if $\mathcal{C}$ satisfies:

1. $\emptyset \notin \mathcal{C}$;

2. if $C_{1}, C_{2} \in \mathcal{C}$ and $C_{1} \subseteq C_{2}$, then $C_{1}=C_{2}$;

3. if $C_{1}$ and $C_{2}$ are distinct sets in $\mathcal{C}$ and $e \in C_{1} \cap C_{2}$, then there exists some $C_{3} \in \mathcal{C}$ such that $C_{3} \subseteq\left(C_{1} \cup C_{2}\right) \backslash\{e\}$.

They can also be characterised in terms of bases.

Theorem 3. Given a finite set $E$ and a collection $\mathcal{B} \subseteq 2^{E}$ of subsets of $E$, there exists a matroid $M$ on $E$ that has $\mathcal{C}$ as its collection of bases if and only if $\mathcal{B}$ satisfies:

1. $\mathcal{B} \neq \emptyset$

2. if $B_{1}, B_{2} \in \mathcal{B}$, then, for any $x \in B_{1} \backslash B_{2}$, there exists some $y \in B_{2} \backslash B_{1}$ such that $\left(B_{1} \backslash\{x\}\right) \cup\{y\}$ is a member of $\mathcal{B}$.

For each of these matroid axiom systems, there exists a polynomial-time algorithm to certify whether a given input describes a matroid according to 
that axiom system, where the input is considered to be a list of sets. Note that it is possible for these collections of subsets of the ground set to be exponential in $|E|$. For example, for any finite set $E$, there is a matroid that has the power set of $E$ as its independent sets.

Example 4. Let $E$ be a finite set and $\mathcal{I} \subseteq 2^{E}$. Then an algorithm for determining whether there is a matroid on $E$ that has $\mathcal{I}$ as its collection of independent sets would consist of:

1. checking that $\mathcal{I}$ is nonempty;

2. for all sets $X \in \mathcal{I}$, checking that removing any element from $X$ returns another set in $\mathcal{I}$; and

3. for all pairs of sets $X, Y \in \mathcal{I}$, checking whether one is larger than the other, and, if so, checking whether any of the elements of the larger set can be added to the smaller to return a set in $\mathcal{I}$.

All of these will be polynomial-time operations in $|\mathcal{I}|$.

Example 5. Let $E$ be a finite set and $\mathcal{C} \subseteq 2^{E}$. Then an algorithm for determining whether there is a matroid on $E$ that has $\mathcal{C}$ as its collection of circuits would consist of:

1. checking that the empty set is not a member of $\mathcal{C}$;

2. for any $C_{1} \in \mathcal{C}$, checking that there is no $C_{2} \in \mathcal{C}$ such that $C_{1} \neq C_{2}$ and $C_{1} \subseteq C_{2}$; and

3. for all pairs of distinct sets $C_{1}, C_{2} \in \mathcal{C}$, checking that, for every $e \in$ $C_{1} \cap C_{2}$, there is some $C_{3} \in \mathcal{C}$ such that $C_{3} \subseteq\left(C_{1} \cup C_{2}\right) \backslash\{e\}$.

All of these will be polynomial-time operations in $|\mathcal{C}|$.

Example 6 . Let $E$ be a finite set and $\mathcal{B} \subseteq 2^{E}$. Then an algorithm for determining whether there is a matroid on $E$ that has $\mathcal{B}$ as its collection of bases would consist of: 


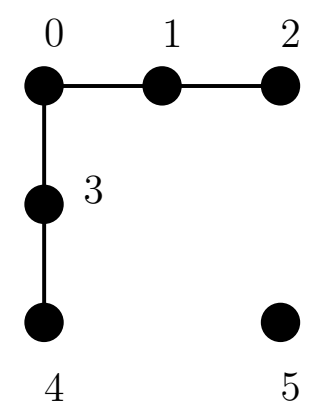

Figure 1.6.1: A rank-three matroid

1. checking that $\mathcal{B}$ is nonempty;

2. for all pairs $B_{1}, B_{2} \in \mathcal{B}$, checking that, for every $x \in B_{1} \backslash B_{2}$, there is some $y \in B_{2} \backslash B_{1}$ such that $\left(B_{1} \backslash\{x\}\right) \cup\{y\}$ is a member of $\mathcal{B}$.

All of these will be polynomial-time operations in $|\mathcal{B}|$.

\subsection{Cyclic flats}

A cyclic flat of a matroid is a (possibly empty) flat that is a union of circuits. For example, the cyclic flats of the matroid in Figure 1.6.1 on page 6 are $\emptyset,\{0,1,2\},\{0,3,4\},\{0,1,2,3,4,5\}$.

Theorem 7. Given a matroid $M$, the cyclic flats $\mathcal{Z}(M)$ of $M$ form a lattice under inclusion.

In this lattice, the join of two cyclic flats $X$ and $Y$ will be the closure in $M$ of $X \cup Y$, and their meet will be the intersection of $X$ and $Y$, with every element that is not in a circuit of $M$ contained in $X \cap Y$ removed. Every finite lattice $\mathcal{Z}$ has a least element that shall be denoted here by $0_{\mathcal{Z}}$. Bonin and de Mier (in a rediscovery of a theorem first proved by Julie Sims in [6]) established a set of axioms that characterise when a collection of subsets $\mathcal{Z}$ of a ground set $E(\mathcal{Z})$, and a rank function $r: \mathcal{Z} \rightarrow \mathbb{Z}_{\geq 0}$ are the ranked cyclic flats of a matroid [1]. 

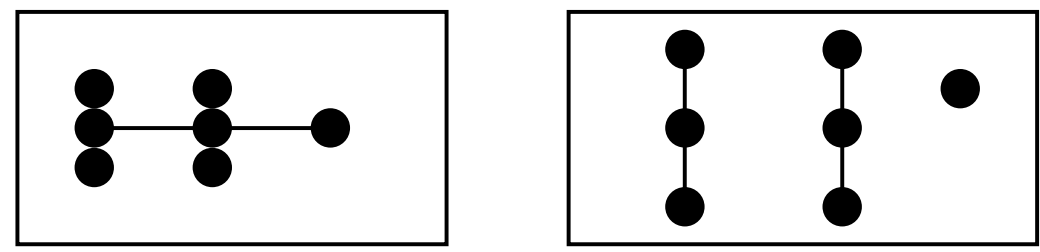

Figure 1.6.2: Two matroids with identical sets of cyclic flats.

Theorem 8. Given a collection $\mathcal{Z}$ of subsets of some ground set $E$ and a function $r: \mathcal{Z} \rightarrow \mathbb{Z}_{\geq 0}$, there is a matroid $M$ on $E$ that has $\mathcal{Z}$ as its lattice of cyclic flats, and $r$ the ranks of those cyclic flats, if and only if $(Z 0) \mathcal{Z}$ is a lattice under inclusion;

(Z1) $r\left(0_{\mathcal{Z}}\right)=0$;

(Z2) $0<r(Y)-r(X)<|Y-X|$ for all $X, Y \in \mathcal{Z}$ with $X \subsetneq Y$; and

(Z3) for all $X, Y \in \mathcal{Z}$,

$$
r(X)+r(Y) \geq r(X \wedge Y)+r(X \vee Y)+|(X \cap Y)-(X \wedge Y)|
$$

The binary operations $\wedge$ and $\vee$ here are the meet and join operations on elements of a lattice (elaborated upon in Chapter 2).

If a rank function $r$ complies with these axioms, we say that it is matroidal. Note that if $X \subseteq Y$, then, as $X \cap Y=X \wedge Y=X$ and $X \vee Y=Y$, the fourth axiom is simply the trivial statement that $r(X)+r(Y) \geq r(X)+r(Y)$.

A collection of subsets itself does not define a matroid, as there may be multiple rank functions that comply with this axiom system. For example, the matroids in Figure 1.6.2 on page 7 have the same cyclic flats, but with different ranks assigned to them.

It is also possible for a collection of sets not to give rise to any matroidal rank functions:

Example 9. Let $\mathcal{Z}=\{\emptyset,\{1\}\}$. For a matroidal rank function $r$, the second cyclic flat axiom requires that $r(\emptyset)=0$. Then by $(Z 2), 0<r(\{1\})<1$, which is impossible, as $r$ takes integer values. 
Example 10. Let

$$
\mathcal{Z}=\{\emptyset,\{1,2\},\{1,2,3,4\},\{5,6\}\{1,2,3,4,5,6\}\}
$$

For a matroidal rank function $r$, the third cyclic flat axiom requires that $r(\{1,2\})=1, r(\{1,2,3,4\})=2, r(\{5,6\})=1$, and $r(\{1,2,3,4,5,6\})=3$. But this contradicts the fourth axiom, as

$$
r(\{1,2\})+r(\{5,6\})=2<3=r(\emptyset)+r(\{1,2,3,4,5,6\}) .
$$

As with the other axiom schemes, there exists a polynomial-time algorithm that will certify whether a triple $(E, \mathcal{Z}, r)$ describes a matroid in accordance with the cyclic flat axioms.

Lemma 11 . Given a partially ordered set $\mathbb{P}=(P, \leq)$, there exists an algorithm that is polynomial in $|P|$ to show whether $\mathbb{P}$ is a lattice.

Proof. Let $X, Y \in P$. Construct the set $P_{X, Y}=\{A \in P: A \leq X, A \leq Y\}$. For all $Z \in P_{X, Y}$, find if there exists some $W \in P_{X, Y}$ such that $W \not Z Z$. If so, then $Z$ is not the greatest lower bound of $X$ and $Y$. If there exists no greatest lower bound, then $\mathbb{P}$ is not a lattice. Use a similar process to find a least upper bound. Repeat over all pairs $X, Y \in P$. This is quartic in $|P|$.

Lemma 12. Given a finite set $E$, a collection of subsets $\mathcal{Z} \subseteq 2^{E}$, and a function $r: \mathcal{Z} \rightarrow \mathbb{Z}_{\geq 0}$, there exists an algorithm that is polynomial in $|\mathcal{Z}|$ to show whether there exists a matroid $M$ on $E$ that has $\mathcal{Z}$ as its cyclic flats, and $r$ as the rank function of $M$ on those cyclic flats.

Proof. Whether $\mathcal{Z}$ is a lattice can be tested in polynomial time, by Lemma 11 above. Note that this involves finding the join and meet of every pair in $\mathcal{Z}$.

For every pair $Z_{1}, Z_{2} \in \mathcal{Z}$, check if $Z_{1} \subsetneq Z_{2}$, and if so, check that $0<$ $r\left(Z_{2}\right)-r\left(Z_{1}\right)<\left|Z_{2}\right|-\left|Z_{1}\right|$. 
For every pair $Z_{1}, Z_{2} \in \mathcal{Z}$, find $Z_{1} \vee Z_{2}$ and $Z_{1} \wedge Z_{2}$, calculate $\left|Z_{1} \cap Z_{2}\right|$ and $\left|Z_{1} \wedge Z_{2}\right|$, and check that

$$
r\left(Z_{1}\right)+r\left(Z_{2}\right) \geq r\left(Z_{1} \vee Z_{2}\right)+r\left(Z_{1} \wedge Z_{2}\right)+\left|Z_{1} \cap Z_{2}\right|-\left|Z_{1} \wedge Z_{2}\right| .
$$

All of these operations are polynomial in $|\mathcal{Z}|$.

This then gives rise to the central question of this thesis.

Problem 13. Given a finite set $E$ and a collection of subsets $\mathcal{Z} \subseteq 2^{E}$, is it possible to determine whether there exists any matroid on $E$ that has $\mathcal{Z}$ as its collection of cyclic flats?

Bonin has conjectured (in personal communication) that there is no finite set of axioms that would characterise sets with matroidal rank functions. We make this formal.

Conjecture 14. There is no finite set of axioms in a formal logical language using

- the subset relation $\subseteq$;

- set intersection $\cap$ and set union $\cup$;

- set cardinality $|\cdot|$;

- the usual order relation $\leq$ on $\mathbb{Z}$;

- arithmetic addition and multiplication;

- logical negation $\neg$;

- $\operatorname{logical}$ conjunction $\wedge$;

- logical implication $\rightarrow$

- the universal and existential quantifiers $\forall$ and $\exists$

that characterises collections of sets that form the cyclic flats of some matroid. 
However, there is another possible method of approaching the problem.

Clearly, if there exists some matroidal $r: \mathcal{Z} \rightarrow \mathbb{Z}_{\geq 0}$, then there is a matroid that has $\mathcal{Z}$ as its cyclic flats.

The cyclic flat axioms $(Z 1),(Z 2)$, and $(Z 3)$ in Theorem 8 are able to be encoded in a system of linear inequalities $A x \leq b$ that define a bounded solution space embedded in $\mathbb{R}^{\mathcal{Z}}$ that contains all matroidal values of $r$.

This is done by representing each set in $\mathcal{Z}$ in a collection of subsets by a column in the matrix, and by noting that, as $r$ must be restricted to integral functions, the following inequalities are implied by the cyclic flat axioms:

$$
\begin{aligned}
r\left(0_{\mathcal{Z}}\right) & \leq 0 \\
-r\left(0_{\mathcal{Z}}\right) & \leq 0 \\
r(Y)-r(X) & \leq|Y|-|X|-1 \\
r(X)-r(Y) & \leq-1 \\
r(W \vee Z)+r(W \wedge Z)-r(W)-r(Z) & \leq|W \wedge Z|-|W \cap Z|
\end{aligned}
$$

for all $X, Y \in \mathcal{Z}$ where $X \subsetneq Y$, and for all $W, Z \in \mathcal{Z}$. A system $A x \leq b$ is constructed by letting the vector $x$ be the (indeterminate) vector of ranks, and by letting each of the above inequalities be a row, where 1 or -1 is placed in the column $V$ of $A$ depending on the sign of $r(V)$ in the relevant inequality, and where the entry in $b$ in that row is simply the right-hand side of that inequality. There is also some simplification of the system by identification of redundancies, as will be explained in Chapter 2.

We label the solution space that arises $P(\mathcal{Z})$, and we call the matrix $A$ of coefficients of this system the constraint matrix of $\mathcal{Z}$, and denote it by $C(\mathcal{Z})$. A face of $P(\mathcal{Z})$ is a subset of $P(\mathcal{Z})$ where one or more of the rows of the system $A x \leq b$ are exact equalities. A minimal face is a face that does not contain any other face, and in a bounded solution space, every minimal face consists of a single point and is called a vertex. Polyhedra are explained in greater detail in Chapter 2.

A matroid's rank function has only integer outputs, and therefore the matroidal rank functions are only those integral points contained within 


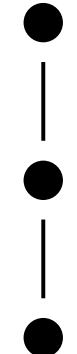

Figure 1.6.3: A lattice $\mathcal{Z}_{1}$

$P(\mathcal{Z})$. To determine the existence of such a rank function on a lattice $\mathcal{Z}$ therefore means determining the existence of an integral point within the solution space. As shown in Chapter 2, the general form of this problem is NP-complete.

However, a subclass of the problem subject to specific constraints may be able to be solved efficiently. For example, there are classes of matrices such that, if a matrix $A$ belongs to such a class, a linear system $A x \leq b$ is guaranteed to have integral vertices. Two such classes are the class of totally unimodular matrices, which are matrices in which every nonsingular submatrix has determinant \pm 1 , and unimodular matrices, which are $m \times n$ matrices in which every nonsingular $m \times m$ submatrix has determinant \pm 1 .

By examination of the nature of the matrix of coefficients $C(\mathcal{Z})$, or the nature of the solution space $P(\mathcal{Z})$, then, we distinguish five classes into which we sort lattices of sets. These five classes are:

Definition 15. A lattice of sets $\mathcal{Z}$ belongs to class I if the constraint matrix $C(\mathcal{Z})$ is totally unimodular.

Example 16. The lattice $\mathcal{Z}_{1}$ in Figure 1.6 .3 on page 11 has the constraint 


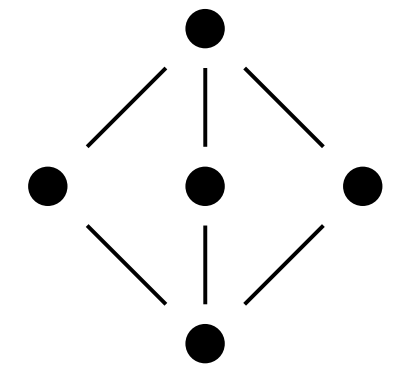

Figure 1.6.4: A lattice $\mathcal{Z}_{2}$

matrix

$$
C\left(\mathcal{Z}_{1}\right)=\left(\begin{array}{ccc}
1 & 0 & 0 \\
-1 & 0 & 0 \\
1 & -1 & 0 \\
-1 & 1 & 0 \\
0 & 1 & -1 \\
0 & -1 & 1
\end{array}\right)
$$

which is totally unimodular.

Definition 17. A lattice of sets $\mathcal{Z}$ belongs to class II if $C(\mathcal{Z})^{T}$ is unimodular.

Example 18. The lattice $\mathcal{Z}_{2}$ in Figure 1.6 .4 on page 12 has the constraint 
matrix

$$
C\left(\mathcal{Z}_{2}\right)=\left(\begin{array}{ccccc}
1 & 0 & 0 & 0 & 0 \\
-1 & 0 & 0 & 0 & 0 \\
1 & -1 & 0 & 0 & 0 \\
-1 & 1 & 0 & 0 & 0 \\
1 & 0 & -1 & 0 & 0 \\
-1 & 0 & 1 & 0 & 0 \\
1 & 0 & 0 & -1 & 0 \\
-1 & 0 & 0 & 1 & 0 \\
0 & 1 & 0 & 0 & -1 \\
0 & -1 & 0 & 0 & 1 \\
0 & 0 & 1 & 0 & -1 \\
0 & 0 & -1 & 0 & 1 \\
0 & 0 & 0 & 1 & -1 \\
0 & 0 & 0 & -1 & 1 \\
1 & -1 & -1 & 0 & 1 \\
1 & -1 & 0 & -1 & 1 \\
1 & 0 & -1 & -1 & 1
\end{array}\right)
$$

and $C\left(\mathcal{Z}_{2}\right)^{T}$ is unimodular.

Definition 19. A lattice of sets $\mathcal{Z}$ belongs to class III if either every vertex of $P(\mathcal{Z})$ is integral or $P(\mathcal{Z})$ is empty.

Example 20. The lattice $\mathcal{Z}_{3}$ in Figure 1.6 .5 on page 14 has all integral vertices Definition 21. A lattice of sets $\mathcal{Z}$ belongs to class IV if either $P(\mathcal{Z})$ has at least one integral vertex, or is empty.

Example 22. The lattice $\mathcal{Z}_{4}$ in Figure 1.6 .6 on page 14 generates the polyhedron $P\left(\mathcal{Z}_{4}\right)$ which has 11 vertices, 8 of which are integral.

Definition 23. A lattice of sets $\mathcal{Z}$ belongs to class $\mathrm{V}$ if $P(\mathcal{Z})$ either contains an integral point, or is empty. 


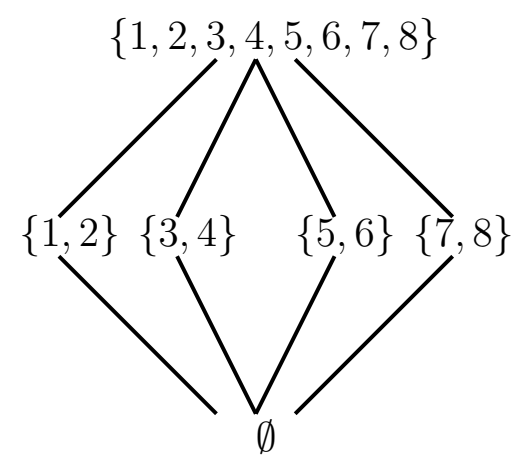

Figure 1.6.5: A lattice $\mathcal{Z}_{3}$ of sets

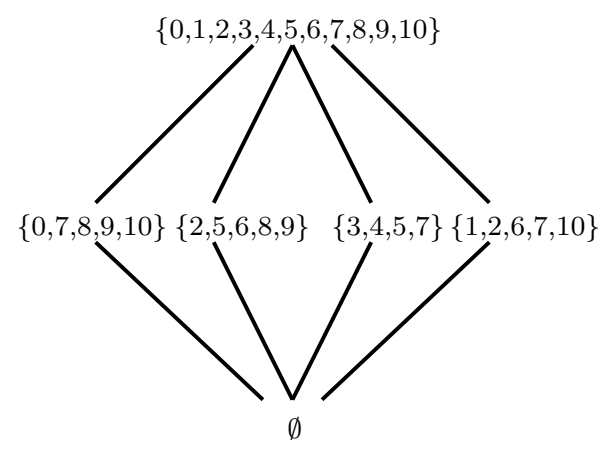

Figure 1.6.6: A lattice $\mathcal{Z}_{4}$ of sets 
Lemma 24. Class I is properly contained in class II

Proof. A lattice of sets $\mathcal{Z}$ is in class I if it has a totally unimodular constraint matrix; total unimodularity implies unimodularity, therefore every class I lattice belongs to class II. The lattice $\mathcal{Z}_{2}$ in Figure 1.6 .4 on page 12 is a lattice that is in class II, but $C\left(\mathcal{Z}_{2}\right)$ contains the submatrix (visible in the final three rows)

$$
\left(\begin{array}{ccc}
-1 & -1 & 0 \\
-1 & 0 & -1 \\
0 & -1 & -1
\end{array}\right)
$$

which has determinant -2 , therefore $C\left(\mathcal{Z}_{2}\right)$ is not totally unimodular. Hence $\mathcal{Z}_{2}$ is not class I, and therefore class I is properly contained in class II.

Lemma 25. Class II is properly contained in class III.

Proof. By Cramer's rule (see Chapter 2), if the matrix of coefficients in a system of linear inequalities is unimodular, then all vertices in the solution space are integral, therefore all class II lattices belong to class III. The lattice $\mathcal{Z}_{3}$ in Figure 1.6 .5 on page 14 is a lattice that is in class III, but it has the $6 \times 6$ submatrix

$$
\left(\begin{array}{cccccc}
-1 & 1 & 0 & 0 & 0 & 0 \\
1 & 0 & -1 & -1 & 0 & 1 \\
1 & 0 & -1 & 0 & -1 & 1 \\
1 & 0 & 0 & -1 & -1 & 1 \\
0 & -1 & 0 & 0 & 0 & 1 \\
1 & 0 & 0 & 0 & 0 & 0
\end{array}\right)
$$

which has determinant -2 , therefore $C\left(\mathcal{Z}_{3}\right)$ is not unimodular. Hence $\mathcal{Z}_{3}$ is not in class II, and therefore class II is properly contained in class III.

Lemma 26. Class III is properly contained in class IV.

Proof. Trivially, if every vertex of $P(\mathcal{Z})$ is integral, then at least one is integral, so class III is contained in class IV. The lattice $\mathcal{Z}_{4}$ in Figure 1.6.6 on page 14 has 8 integral vertices and 3 non-integral vertices, therefore it 
belongs to class IV, but not to class III. So class III is properly contained in class IV.

Lemma 27. Class IV is contained in class V.

Proof. If $P(\mathcal{Z})$ has at least one integral vertex, then it has at least one integral point, therefore class IV is contained in class $\mathrm{V}$.

Experimental trials have returned a number of lattices in which the solution space has at least one non-integral vertex, but in every such case, all vertices have been half-integral. As a result, we conjecture that this is true of all lattices.

Conjecture 28. Given a lattice of sets $\mathcal{Z}$, if $P(\mathcal{Z})$ is nonempty, then every entry in a vertex of $P(\mathcal{Z})$ belongs to $\left\{\frac{z}{2}: z \in \mathbb{Z}\right\}$.

Experimentation has not to date produced any examples of lattices that belong to class $\mathrm{V}$, but not to class IV. As such, we make the following conjecture.

Conjecture 29. Every lattice of sets belongs to class IV.

If this is the case, then, given that it is possible, in polynomial time, to both construct the system of inequalities and test the resulting polyhedron for nonemptiness, it would mean that for any lattice of sets $\mathcal{Z}$, there exists an algorithm that is polynomial in the size of $\mathcal{Z}$ that can determine whether there is any matroid that has $\mathcal{Z}$ as its lattice of cyclic flats. 


\section{Chapter 2}

\section{Preliminaries}

\subsection{Matroid minors}

Definition 30. If $M$ is a matroid on ground set $E$, then, given some $D \subseteq E$, $M \backslash D$ is the matroid on $E \backslash D$, called the deletion of $D$ from $M$, where $J$ is an independent set of $M \backslash D$ if and only if $J \subseteq E \backslash D$ is an independent set of $M$.

Definition 31. If $M$ is a matroid on ground set $E$, then, given some $D \subseteq E$, $M / D$ is the matroid on $E \backslash D$, called the contraction of $M$ onto $E \backslash D$, given by $\left(M^{*} \backslash D\right)^{*}$.

Definition 32. If $M$ is a matroid, then a minor of $M$ is a matroid of the form $M \backslash D_{1} / D_{2}$, where $D_{1}$ and $D_{2}$ are disjoint subsets of $E(M)$.

\subsection{Lattices}

Definition 33. A partial order is a relation $\leq$ on a set $R$ that satisfies the conditions of:

- reflexivity: $x \leq x$ for all $x \in R$;

- transitivity: if $x \leq y$ and $y \leq z$, then $x \leq z$, for all $x, y, z \in R$; and 
- antisymmetry: if $x \leq y$ and $y \leq x$, then $x=y$, for all $x, y \in R$.

A set with a partial order is called a poset.

Given a set $R$ partially ordered by $\leq$, we write $x<y$ if $x \leq y$ and $x \neq y$. If, for all $x, y \in R$, either $x \leq y$ or $y \leq x$, then $R$ is totally ordered by $\leq$. A totally ordered set, or a totally ordered subset of a poset, is called a chain.

Definition 34. In a poset $(R, \leq), y$ covers $x$ if $x<y$ and there exists no $z \in R$ such that $x<z<y$.

Definition 35. A poset $\mathcal{Z}$ is a lattice if, for every $x, y \in \mathcal{Z}, x$ and $y$ have a unique greatest lower bound (called the meet of $x$ and $y$, and written $x \wedge y$ ) and least upper bound (called the join of $x$ and $y$, written $x \vee y$ ). That is, for every $x$ and $y$ in $\mathcal{Z}$ :

- there exists some $z \in \mathcal{Z}$ such that $z \leq x$ and $z \leq y$, and if $w \leq x$ and $w \leq y$ for any $w \in \mathcal{Z}$, then $w \leq z$; and

- there exists some $p \in \mathcal{Z}$ such that $x \leq p$ and $y \leq p$, and if $x \leq q$ and $y \leq q$ for any $q \in \mathcal{Z}$, then $p \leq q$.

A consequence of this is that a finite lattice $\mathcal{Z}$ has a least element $0_{\mathcal{Z}}$ and a greatest element $1_{\mathcal{Z}}$, i.e., for every $x \in \mathcal{Z}, 0_{\mathcal{Z}} \leq x \leq 1_{\mathcal{Z}}$.

Definition 36. A complete lattice is a lattice $(\mathcal{Z}, \leq)$ such that every $K \subseteq \mathcal{Z}$ has a join $\bigvee K$ and a meet $\bigwedge K$.

Every finite lattice is complete [3].

Definition 37. If $R$ and $S$ are disjoint posets, the linear sum $R \oplus S$ is the poset on $R \cup S$ where $x \leq_{R \oplus S} y$ if and only if $x \leq_{R} y, x \leq_{S} y$, or $x \in R$ and $y \in S$.

Definition 38. If $R$ and $S$ are posets, the Cartesian product $R \times S$ is the poset on $\{(x, y): x \in R, y \in S\}$ where $(x, y) \leq_{R \times S}(z, w)$ if and only if $x \leq_{R} z$ and $y \leq_{S} w$. 
The linear sum of two lattices is a lattice. The Cartesian product of two lattices is a lattice [3].

In this work, lattice shall be taken to mean a lattice of subsets of a ground set $E$, ordered by set inclusion, unless otherwise specified.

\subsection{Linear and integer programming}

Definition 39. A set $P \in \mathbb{R}^{n}$ is called a polyhedron if $P=\{x: A x \leq b\}$ for some $m \times n$ matrix $A$ and vector $b$.

A polyhedron $P$ is bounded if there exists some $M \in \mathbb{N}$ such that, for all $z \in P,|z| \leq M$. A bounded polyhedron is called a polytope.

Definition 40. A face of a polyhedron $P$ is a subset $F \subseteq P$ where $F=$ $\left\{x: A x \leq b, A^{\prime} x=b^{\prime}\right\}$, where $A^{\prime}$ is a $\ell \times n$ submatrix of $A$, and $b^{\prime}$ is the vector consisting of the corresponding rows from $b$. If $A^{\prime}$ is a nonsingular $n \times n$ matrix, then $R$ is a single point and is called a vertex of $P$.

Linear programming refers to the problem of optimising a linear functional over a polyhedron. That is, given a cost vector $c$ and a polyhedron $P=\{x: A x \leq b\}$, maximise (or minimise) $c x$ while $x \in P$.

Integer programming is linear programming with the additional constraint that $x$ is integral, i.e., $x \in \mathbb{Z}^{n}$.

Lemma 41 (Cramer's rule). Given a linear system of equalities $A x=b$, where $A$ is a nonsingular square matrix, the entries in the solution $x$ are given by

$$
x_{i}=\frac{\operatorname{det}\left(A_{i}\right)}{\operatorname{det}(A)}
$$

where $A_{i}$ is obtained by replacing the $i$ th column of $A$ with $b[5]$.

Proposition 42. The problem of determining whether an integral solution to $A x \leq b$ exists is NP-complete. 
Proof. If $A$ is an $m \times n$ matrix, then to verify a candidate solution $z$, simply perform the matrix multiplication $A z$, which involves $m n$ arithmetic operations, and compare this result to the vector $b$ to check that $A z \leq b$. Therefore the problem is in NP.

That the problem is NP-complete can then be shown by reduction from the Boolean 3-satisfiability problem. Given a Boolean expression $X$ in conjunctive normal form with $n$ variables $x_{1}, \ldots, x_{n}$ and $m$ clauses, where every clause has at most three literals, construct an $(m+2 n) \times n$ matrix $A$. For $1 \leq i \leq m$, in the $i$ th row of $A$, let the $j$ th entry be 1 if $x_{j}$ is in the $i$ th clause of $X,-1$ if $\neg x_{j}$ is in the $i$ th clause of $X$, and 0 otherwise. Then for $1 \leq k \leq n$, let the $(m+2 k-1)$ th row have 1 in the $k$ th column, and the $(m+2 k)$ th row have -1 in the $k$ th column.

Then construct a column vector $b$ by letting the $i$ th row of $b$ be $1-\ell$, where $\ell$ is the number of negative literals in the $i$ th clause of $X$, for all $1 \leq i \leq m$, and by letting the $(m+2 k-1)$ th row be 1 and the $(m+2 k)$ th row be 0 for all $1 \leq k \leq n$.

For example, if the Boolean expression is

$$
X=\left(x_{1} \vee \neg x_{2} \vee \neg x_{3}\right) \wedge\left(x_{2} \vee \neg x_{3} \vee x_{4}\right) \wedge\left(\neg x_{1} \vee x_{4} \vee x_{5}\right)
$$

then the system of inequalities constructed by this method shall be 


$$
\left(\begin{array}{ccccc}
1 & -1 & -1 & 0 & 0 \\
0 & 1 & -1 & 1 & 0 \\
-1 & 0 & 0 & 1 & 1 \\
1 & 0 & 0 & 0 & 0 \\
-1 & 0 & 0 & 0 & 0 \\
0 & 1 & 0 & 0 & 0 \\
0 & -1 & 0 & 0 & 0 \\
0 & 0 & 1 & 0 & 0 \\
0 & 0 & -1 & 0 & 0 \\
0 & 0 & 0 & 1 & 0 \\
0 & 0 & 0 & -1 & 0 \\
0 & 0 & 0 & 0 & 1 \\
0 & 0 & 0 & 0 & -1
\end{array}\right)\left(\begin{array}{l}
x_{1} \\
x_{2} \\
x_{3} \\
x_{4} \\
x_{5}
\end{array}\right) \leq\left(\begin{array}{c}
-1 \\
0 \\
0 \\
1 \\
0 \\
1 \\
0 \\
1 \\
0 \\
1 \\
0 \\
1 \\
0
\end{array}\right) .
$$

To understand this construction, note that, if the Boolean values of True and False are interpreted as being numerically 1 and 0 , respectively, the following equivalences hold:

$$
\begin{aligned}
x_{1} \vee x_{2} \vee x_{3} & \Leftrightarrow x_{1}+x_{2}+x_{3} \geq 1 \\
x_{1} \vee x_{2} \vee \neg x_{3} & \Leftrightarrow x_{1}+x_{2}+\left(1-x_{3}\right) \geq 1 \\
x_{1} \vee \neg x_{2} \vee x_{3} & \Leftrightarrow x_{1}+\left(1-x_{2}\right)+x_{3} \geq 1 \\
x_{1} \vee \neg x_{2} \vee \neg x_{3} & \Leftrightarrow x_{1}+\left(1-x_{2}\right)+\left(1-x_{3}\right) \geq 1 \\
\neg x_{1} \vee x_{2} \vee x_{3} & \Leftrightarrow\left(1-x_{1}\right)+x_{2}+x_{3} \geq 1 \\
\neg x_{1} \vee x_{2} \vee \neg x_{3} & \Leftrightarrow\left(1-x_{1}\right)+x_{2}+\left(1-x_{3}\right) \geq 1 \\
\neg x_{1} \vee \neg x_{2} \vee x_{3} & \Leftrightarrow\left(1-x_{1}\right)+\left(1-x_{2}\right)+x_{3} \geq 1 \\
\neg x_{1} \vee \neg x_{2} \vee \neg x_{3} & \Leftrightarrow\left(1-x_{1}\right)+\left(1-x_{2}\right)+\left(1-x_{3}\right) \geq 1 .
\end{aligned}
$$

Therefore, a vector $z$ that solves $A z \geq b$ (or, equivalently, $-A z \leq-b$ ) that has only 0 and 1 as entries will also give an assignment of values to the Boolean variables that satisfies the expression $X$. Rows $m+1$ through $m+2 n$ restrict each variable to being between 0 and 1 , and so requiring 
that solutions be integral gives such a satisfying assignment. So solving this integer programming problem would solve 3-satisfiability, therefore integer programming is NP-hard, and therefore it is NP-complete.

The proof of NP-completeness is adapted from [4].

\subsection{Matrices}

Definition 43. A matrix $A$ is totally unimodular if every nonsingular square submatrix of $A$ has determinant \pm 1 .

Proposition 44. A matrix $A$ is totally unimodular if and only if for every subset $\left\{Y_{1}, Y_{2}, \ldots, Y_{t}\right\}$ of columns of $A$, there exists some sequence $\left(\epsilon_{j}\right)_{j \leq t}$, where $\epsilon_{j}= \pm 1$ for all $1 \leq j \leq t$, such that the vector $\sum_{i=1}^{t} \epsilon_{i} Y_{i}$ has all entries in $\{-1,0,1\}$ (i.e., it is a totally unimodular vector) [5].

Definition 45. An $m \times n$ matrix $A$ with $m<n$ is unimodular if every nonsingular $m \times m$ submatrix of $A$ has determinant \pm 1 .

Definition 46. A basis of a matrix $A$ of rank $m$ is a submatrix of $A$ made up of $m$ linearly independent columns of $A$.

Proposition 47. A matrix $A$ is unimodular if and only if there exists some basis $B$ of $A$ such that $B$ is unimodular and the unique matrix $C$ satisfying $B C=A$ is totally unimodular [5].

\subsection{Cyclic flat axioms as a system of inequalities}

Proposition 48. Given a lattice of sets $\mathcal{Z}$, the cyclic flat axioms $(Z 1),(Z 2),(Z 3)$ can be represented as a system of inequalities $A x \leq b$.

Proof. Construct the matrix $A$ and vector $b$ as follows:

1. Let each of the $n$ elements of the lattice be represented by a column of the matrix. 
2. Add a row:

(a) with 1 in the column $0_{\mathcal{Z}}$ and 0 in all other columns, and let that row of $b$ be 0 ; and a row

(b) with -1 in the column $0_{\mathcal{Z}}$ and 0 in all other columns, and let that row of $b$ be 0 .

3. For all $X, Y \in \mathcal{Z}$ with $X \subsetneq Y$,

(a) add a row with 1 in the column $Y,-1$ in the column $X$, and 0 in all other columns, and let that row of $b$ be $|Y-X|-1$; and

(b) add a row with -1 in the column $Y, 1$ in the column $X$, and 0 in all other columns, and let that row of $b$ be -1 .

4. For all $X, Y \in \mathcal{Z}$ where $X$ and $Y$ are incomparable, add a row with -1 in the columns $X$ and $Y, 1$ in the columns $X \wedge Y$ and $X \vee Y$, and 0 in all other columns, and let that row of $b$ be $-|(X \cap Y)-(X \wedge Y)|$.

As this is a system of linear inequalities, there is a polyhedron $P(\mathcal{Z}) \subseteq \mathbb{R}^{\mathcal{Z}}$ that it defines. Note that the rank of $0_{\mathcal{Z}}$ is fixed at 0 , the rank of $1_{\mathcal{Z}}$ is at most $\left|1_{\mathcal{Z}}\right|-1$, and the rank of every other element must fall between these two. Therefore $P(\mathcal{Z})$ is bounded.

The polyhedron $P(\mathcal{Z})$ consists of all points in $\mathbb{R}^{\mathcal{Z}}$ satisfying $A x \leq b$. Therefore each point in $P(\mathcal{Z})$ satisfies the cyclic flat axioms $(Z 1),(Z 2)$, and (Z3). As matroid rank functions must be integral, then only those integral points in $P(\mathcal{Z})$ are valid rank functions on $\mathcal{Z}$. So then to find a matroidal rank function for $\mathcal{Z}$ is an integer programming problem. However, as has been shown, integer programming is NP-complete, so finding solutions may not be computationally feasible.

To approach the problem, first note that the matrix $A$ is highly redundant. In particular, if $X \subsetneq Y \subsetneq Z$, then there are rows specifying: 


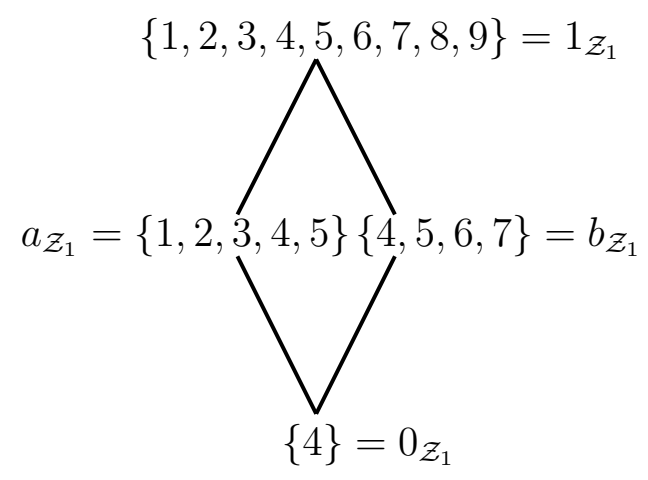

Figure 2.5.1: A lattice $\mathcal{Z}_{1}$

1. that $r(Y)-r(X) \geq 1, r(Z)-r(Y) \geq 1$, and $r(Z)-r(X) \geq 1$, the third of which is implied by the first two; and

2. that $r(Y)-r(X)<|Y-X|, r(Z)-r(Y)<|Z-Y|$, and $r(Z)-$ $r(X)<|Z-X|=|Z-Y|+|Y-X|$, the third of which is again implied by the first two.

So if a point $p \in \mathbb{R}^{\mathcal{Z}}$ satisfies the first two inequalities in either case above, it must also satisfy the third. So then all such redundancies can be removed if all rows added in step 3 above are removed except where $Y$ covers $X$. Call this irredundant matrix $C(\mathcal{Z})$. As only those rows were removed which were logically implied by the remaining rows, $C(\mathcal{Z}) x \leq b$ has the same solution space as $A x \leq b$.

Example 49. Consider the lattice $\mathcal{Z}_{1}$ in Figure 2.5.1 on page 24. The system 
of inequalities constructed from this will be

$$
\begin{aligned}
& \begin{array}{lllll}
0_{\mathcal{Z}_{1}} & a_{\mathcal{Z}_{1}} & b_{\mathcal{Z}_{1}} & 1_{\mathcal{Z}_{1}} & b
\end{array} \\
& \left(\begin{array}{cccc}
1 & 0 & 0 & 0 \\
-1 & 0 & 0 & 0 \\
-1 & 1 & 0 & 0 \\
1 & -1 & 0 & 0 \\
-1 & 0 & 1 & 0 \\
1 & 0 & -1 & 0 \\
0 & -1 & 0 & 1 \\
0 & 1 & 0 & -1 \\
0 & 0 & -1 & 1 \\
0 & 0 & 1 & -1 \\
1 & -1 & -1 & 1
\end{array}\right)\left(\begin{array}{l}
r_{1} \\
r_{2} \\
r_{3} \\
r_{4}
\end{array}\right) \leq\left(\begin{array}{c}
0 \\
0 \\
3 \\
-1 \\
2 \\
-1 \\
3 \\
-1 \\
4 \\
-1 \\
-1
\end{array}\right)
\end{aligned}
$$

A constraint matrix $C(\mathcal{Z})$ constructed in this manner will always contain exactly 2 rows of weight 1 (rows having a single nonzero entry), which specify that the rank of the least element is 0 ; a number of rows of weight 2 (rows having two nonzero entries), corresponding to cover relations in $\mathcal{Z}$; and a number of rows of weight 4 (rows having four nonzero entries), corresponding to incomparable pairs in $\mathcal{Z}$.

The integer programming problem reduces to a linear programming problem if the convex hull of all integral solutions to $C(\mathcal{Z}) x \leq b$ is equal to the entire set of solutions, i.e., if the polyhedron $P=\left\{x \in \mathbb{R}^{n}: C(\mathcal{Z}) x \leq b\right\}$ has integral vertices.

There are certain special cases where it can be proven that this is the case. The vertices of the polyhedron correspond to full-rank submatrices of $C$, i.e., a vertex of $P$ is the unique solution $x$ to $C^{\prime} x=b^{\prime}$ for some nonsingular $n \times n$ submatrix $C^{\prime}$ of $C$. By Cramer's rule, the elements of $x$ are

$$
x_{i}=\frac{\operatorname{det}\left(C_{i}^{\prime}\right)}{\operatorname{det}\left(C^{\prime}\right)} .
$$

Clearly, if all nonsingular, square submatrices of $C$ had determinant \pm 1 (i.e., $C$ is totally unimodular), then $x$ would be integral, and hence all vertices 
of $P$ would be integral. Indeed, it is only required that nonsingular $n \times n$ submatrices have determinant \pm 1 (i.e., that $C^{T}$ be unimodular).

This is the basis for the five classes described in Chapter 1.

\subsection{Elementary results concerning cyclic flats}

Lemma 50. The circuits of $M \backslash e$ are the circuits of $M$ that do not contain $e$.

Proof. If $X$ is independent in $M \backslash e$, then $X$ is independent in $M$. If $C$ is dependent in $M \backslash e$, then $C$ is dependent in $M$, and, furthermore, $e \notin C$.

Lemma 51. If $F$ is a cyclic flat of $M \backslash e$, then exactly one of $F$ and $F \cup\{e\}$ is a cyclic flat of $M$.

Proof. Let $F$ be a cyclic flat of $M \backslash e$. If $F$ is not a flat of $M$, then $F \neq$ $\mathrm{cl}_{M}(F)$, and hence there exists some $x$ such that $x \in \operatorname{cl}_{M}(F)-F$. Therefore, there exists some circuit $C$ such that $x \in C \subseteq F \cup\{x\}$. Suppose that $x \neq e$; then, as $x \in \operatorname{cl}_{M}(F), r(F)=r(F \cup\{x\})$, hence $F$ is not a flat of $M \backslash e$. This is a contradiction. Therefore, $x=e$, meaning that $\operatorname{cl}_{M}(F)=F \cup\{e\}$. So, if $F$ is not a flat of $M$, then $F \cup\{e\}$ is.

By Lemma 50, if $F$ is a union of circuits of $M \backslash e$, then $F$ is a union of circuits of $M$. If $F$ is not a flat of $M$, then the flat $F \cup\{e\}$ of $M$ contains a circuit $C$ such that $e \in C \subseteq F \cup\{e\}$. So either $F$ or $F \cup\{e\}$ is a cyclic flat of $M$.

Lemma 52. Let $\mathcal{Z}$ be a lattice of sets and $C(\mathcal{Z})$ its constraint matrix. Then every row basis of $C(\mathcal{Z})$ contains a row of weight 1 .

Proof. Suppose otherwise. Then there is some collection $X=\left\{X_{1}, X_{2}, \ldots, X_{n}\right\}$ of rows of $C(\mathcal{Z})$ such that

$$
a_{1} X_{1}+a_{2} X_{2}+\cdots+a_{n} X_{n}=\left[\begin{array}{llll}
1 & 0 & \cdots & 0
\end{array}\right]
$$

and where $\mathrm{X}$ does not contain either of the rows of weight 1 . 
The entries of this vector obviously must sum to 1 . But the sum of the entries in each individual vector $X_{i}$ sums to 0 . By the commutativity of addition, this is a contradiction. Therefore every row basis of $C(\mathcal{Z})$ contains a row of weight 1 . 


\section{Chapter 3}

\section{Special classes of lattices}

\subsection{Loops and coloops}

Lemma 53. If $\mathcal{Z}$ is a matroidal lattice of sets with ground set $E(\mathcal{Z})$, then, if we let $x$ be an element not in $E(\mathcal{Z})$,

$$
\mathcal{Z}^{\prime}=\{X \cup\{x\}: X \in \mathcal{Z}\}
$$

is a matroidal lattice.

Proof. Let $M$ be a matroid on $E(\mathcal{Z})$ that has $\mathcal{Z}$ as its lattice of cyclic flats. Clearly, $\mathcal{Z}^{\prime}$ is isomorphic to the lattice of cyclic flats of $M \oplus U_{0,1}$, the matroid $M$ extended by a loop element, and $r_{M \oplus U_{0,1}}(X \cup\{x\})=r_{M}(X)$ for all $X \in \mathcal{Z}$.

Lemma 54. If $\mathcal{Z}$ is a matroidal lattice of sets with ground set $E(\mathcal{Z})$ and $x \in 0_{\mathcal{Z}}$, then

$$
\mathcal{Z}^{\prime}=\{X \backslash\{x\}: X \in \mathcal{Z}\}
$$

is a matroidal lattice.

Proof. Let $M$ be a matroid on $E(\mathcal{Z})$ that has $\mathcal{Z}$ as its lattice of cyclic flats. $0_{\mathcal{Z}}$ contains $x$, therefore $r_{M}(\{x\})=0$. So $x$ is a loop in $M$. Clearly, $\mathcal{Z}^{\prime}$ is the lattice of cyclic flats of $M \backslash x$, and $r_{M \backslash x}(X \backslash\{x\})=r_{M}(X)$ for all $X \in \mathcal{Z}$. 
Lemma 55. If $\mathcal{Z}$ is a matroidal lattice of sets with ground set $E(\mathcal{Z})$, then $\mathcal{Z}$ is also the lattice of cyclic flats of a matroid with ground set $E(\mathcal{Z}) \cup\{x\}$, where $x \notin E(\mathcal{Z})$.

Proof. As none of the cyclic flat axioms make reference to the ground set of the lattice $\mathcal{Z}$, this follows directly from the fact that $\mathcal{Z}$ is matroidal. It is trivial to see that if $\mathcal{Z}$ is the lattice of cyclic flats of a matroid $M$ on $E(\mathcal{Z})$, then $\mathcal{Z}$ is also the lattice of cyclic flats of the matroid $M \oplus U_{1,1}$, the matroid $M$ extended by a coloop, and that $r_{M \oplus U_{1,1}}(X)=r_{M}(X)$ for all $X \in \mathcal{Z}$.

Lemma 56. If $\mathcal{Z}$ is a matroidal lattice of sets with ground set $E(\mathcal{Z})$, and $x \in E(\mathcal{Z}) \backslash 1_{\mathcal{Z}}$, then $\mathcal{Z}$ is also the lattice of cyclic flats of a matroid with ground set $E(\mathcal{Z}) \backslash\{x\}$.

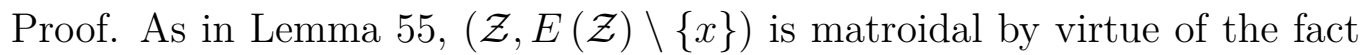
that $(\mathcal{Z}, E(\mathcal{Z}))$ is, the cyclic flat axioms not referring to the ground set. Furthermore, if $M$ is a matroid on $E(\mathcal{Z})$ with lattice of cyclic flats $\mathcal{Z}$, then $x$ is a coloop in $M$, and $\mathcal{Z}$ is clearly also the lattice of cyclic flats of the matroid $M \backslash x$, where $r_{M \backslash x}(X)=r_{M}(X)$ for all $X \in \mathcal{Z}$.

Definition 57. By analogy with matroids, as shown in Lemmas 53-56, given a lattice of sets $\mathcal{Z}$ with ground set $E(\mathcal{Z})$, call an element $x$ a loop if $x \in 0_{\mathcal{Z}}$ and a coloop if $x \in E(\mathcal{Z}) \backslash 1_{\mathcal{Z}}$.

The purpose of this definition is to simplify the following work. Having shown that loops and coloops can be freely added to or removed from a lattice of sets without changing the ranks of the cyclic flats, it shall be assumed for simplicity that the least element $0_{\mathcal{Z}}$ is the empty set, and the greatest element $1_{\mathcal{Z}}$ is the ground set $E(\mathcal{Z})$ of the lattice of sets.

\subsection{Lattices of height 3}

Definition 58. The height of a lattice $\mathcal{Z}$ is the size of the largest chain in $\mathcal{Z}$. 
Lemma 59. Let $\mathcal{Z}$ be a lattice of sets where every maximal chain has 3 elements. If the polyhedron $P(\mathcal{Z})$ is nonempty, then it has an integral vertex.

Proof. Every pair of elements in $\mathcal{Z}$ aside from $0_{\mathcal{Z}}$ and $1_{\mathcal{Z}}$ will be incomparable.

Let $X$ and $Y$ be incomparable sets in $\mathcal{Z}$. Note that, necessarily, $Y \neq$ $E(\mathcal{Z})$. By the cyclic flat axioms, a matroidal rank function $r$ must satisfy

$$
\begin{aligned}
r(X)+r(Y) & \geq r(X \vee Y)+r(X \wedge Y)+|(X \cap Y) \backslash(X \wedge Y)| \\
& =r(E(\mathcal{Z}))+r(\emptyset)+|X \cap Y| \\
& =r(E(\mathcal{Z}))+|X \cap Y|,
\end{aligned}
$$

so

$$
\begin{aligned}
r(X) & \geq|X \cap Y|+r(E)-r(Y) \\
& \geq|X \cap Y|+1 .
\end{aligned}
$$

Therefore, in a lattice with such a structure that has a matroidal rank function, the rank of any set $X$ (other than $0_{\mathcal{Z}}$ and $1_{\mathcal{Z}}$ ) is strictly greater than the size of the intersection of $X$ with any incomparable set.

The rank of $1_{\mathcal{Z}}$ must be strictly greater than the rank of any $X \in \mathcal{Z} \backslash 1_{\mathcal{Z}}$. Therefore

$$
r\left(1_{\mathcal{Z}}\right) \geq \max \left\{|X \cap Y|: X, Y \in \mathcal{Z}, X \neq Y, 0<|X|,|Y|<\left|1_{\mathcal{Z}}\right|\right\}+2 .
$$

So construct a rank function $r$ on $\mathcal{Z}$ where

$$
\begin{aligned}
& r\left(0_{\mathcal{Z}}\right)=0 \\
& r\left(1_{\mathcal{Z}}\right)=\max \left\{|X \cap Y|: X, Y \in \mathcal{Z}, X \neq Y, 0<|X|,|Y|<\left|1_{\mathcal{Z}}\right|\right\}+2 .
\end{aligned}
$$

Let $p$ be the rank of $1_{\mathcal{Z}}$. Then for all $U \in \mathcal{Z} \backslash\left\{0_{\mathcal{Z}}, 1_{\mathcal{Z}}\right\}$, let:

$$
r(U)= \begin{cases}p-1, & \text { if }|U| \geq p, \text { or }|U \cap Y|=p-2 \text { for some } Y \in \mathcal{Z} \\ |U|-1, & \text { otherwise. }\end{cases}
$$

As $\mathcal{Z}$ is a lattice by definition and $r\left(0_{\mathcal{Z}}\right)=0$ by construction, if this is not a matroidal assignment of ranks, either the third or the fourth axiom must be violated. 
As $p \geq 2$, it is clear that $r(U) \geq 1$ for any $U \in \mathcal{Z} \backslash\left\{0_{\mathcal{Z}}\right\}$, unless $|U|=1$. In that case, $r(U) \leq 0$, but $|U|>\left|0_{\mathcal{Z}}\right|$, meaning that $r(U) \geq r\left(0_{\mathcal{Z}}\right)+1=1$, which is a contradiction. There is no solution to these inequalities, meaning that the polyhedron $P(\mathcal{Z})$ is empty.

Note that $r\left(1_{\mathcal{Z}}\right)>r(U)$ for all $U \in \mathcal{Z}$ by construction. Then suppose that there is some pair $X, Y \in \mathcal{Z}$ such that $Y$ covers $X$ and

$$
r(Y)-r(X) \geq|Y|-|X|
$$

By the structure of $\mathcal{Z}$, either $Y=1_{\mathcal{Z}}$ or $X=0_{\mathcal{Z}}$. If $Y=1_{\mathcal{Z}}$ and $r(X)=p-1$, then $|Y|-|X|=1$; in this case, as the cyclic flat axioms would require that $r(Y)-r(X) \leq 0$ and $r(Y)-r(X) \geq 1$, the polyhedron $P(\mathcal{Z})$ is empty. If $Y=1_{\mathcal{Z}}$ and $r(X)=|X|-1$, then, as the rank of $1_{\mathcal{Z}}$ cannot be decreased and the rank of $X$ cannot be increased without violating one of the cyclic flat axioms, there is no solution to the system of inequalities established by the cyclic flat axioms, and so $P(\mathcal{Z})$ is empty.. If $X=0_{\mathcal{Z}}$, then $r(Y)<|Y|$ explicitly by construction. So, if $r$ violates the third cyclic flat axiom, $P(\mathcal{Z})$ is empty.

So suppose the fourth axiom is violated. Then there is some pair $X, Y \in$ $\mathcal{Z}$ such that

$r(X)+r(Y)<r(X \vee Y)+r(X \wedge Y)+|(X \cap Y) \backslash(X \wedge Y)|=r(E)+|X \cap Y|$

Clearly, $X$ and $Y$ must be incomparable, as if they were comparable, their join would be the larger of the two, and the meet and intersection would both be the lesser, which would make the inequality trivially true.

Now suppose that $r(X)=p-1$. Then

$$
p-1+r(Y)<r(E)+|X \cap Y|=p+|X \cap Y|,
$$

SO

$$
r(Y)<|X \cap Y|+1,
$$

which contradicts the fact that $r(Y)$ must be strictly greater than its intersection with any incomparable cyclic flat, as demonstrated earlier. So if 
$r(X)=p-1$ and the fourth axiom is violated, there is no point that satisfies the cyclic flat axioms, and so $P(\mathcal{Z})$ is empty.

Then suppose that $r(X), r(Y)<p-1$. Then $r(X)=|X|-1$ and $r(Y)=|Y|-1$. So neither $X$ nor $Y$ may be assigned a greater rank by any possible point in $P(\mathcal{Z})$. But, as $E$ has the least possible rank that may be assigned by any point in $P(\mathcal{Z})$, the polyhedron must be empty.

So, given such a lattice, the construction above returns a matroidal rank function $r$, if $P(\mathcal{Z})$ is nonempty. As $p$ is an integer, and $|U|$ is an integer for all $U \in \mathcal{Z}, r$ is an integral function.

As $r$ is constructed solely by setting inequalities in the system $C(\mathcal{Z}) x \leq b$ to be equalities, it is a vertex of $P(\mathcal{Z})$.

\subsection{Certain types of lattice of width at most 2}

Definition 60 . The width of a lattice $\mathcal{Z}$ is the size of the largest antichain in $\mathcal{Z}$.

Lemma 61 . If a lattice of sets $\mathcal{Z}$ is a chain, then $C(\mathcal{Z})$ is totally unimodular.

Proof. A chain has no incomparable pairs. Therefore, every row in $C(\mathcal{Z})$ is either a row defining the rank of the least element, or a row corresponding to a cover relation in $\mathcal{Z}$. Therefore, every row has at most two non-zero entries $(+1$ and -1$)$, and, in each row, the sum of any subset of rows will be 0,1 , or -1 .

Therefore, by Proposition 44, $C(\mathcal{Z})$ is totally unimodular.

Lemma 62. If $\mathcal{Z}$ is a lattice of sets, and $X, Y \in \mathcal{Z}$ such that $X \subsetneq Y$ and $|Y|=|X|+1$, then $P(\mathcal{Z})$ is empty.

Proof. Let $r$ be a point in $P(\mathcal{Z})$. Then $r(Y)$ and $r(X)$ obey the inequalities $1 \geq r(Y)-r(X) \leq|Y|-|X|-1=0$. Clearly, this is impossible. Therefore no such point can exist. 
Theorem 63. Let $\mathcal{Z}$ be a lattice of sets of width 2 where if $X$ and $Y$ are incomparable, then $X$ and $Y$ are disjoint, and $X \vee Y=1_{\mathcal{Z}}$. Then $\mathcal{Z}$ belongs to class IV.

Proof. Recursively construct a rank function $r: \mathcal{Z} \rightarrow \mathbb{Z}_{\geq 0}$ by:

$$
r(X)= \begin{cases}0, & \text { if } X=0_{\mathcal{Z}} ; \\ |X|-1, & \text { if } X \text { covers } 0_{\mathcal{Z}} ; \\ r(Y)+1, & \text { if } X \text { covers } Y \neq 0_{\mathcal{Z}} \text { and } X \neq 1_{\mathcal{Z}} \\ \max \{r(Y): Y \leq X\}+1, & \text { if } X=1_{\mathcal{Z}} .\end{cases}
$$

We will show that either $r$ is a vertex of $P(\mathcal{Z})$, or $P(\mathcal{Z})$ is empty.

If $X$ covers $Y$, then if $r(X)-r(Y)=1$ or $r(X)-r(Y)=|X|-|Y|-1$, there is an inequality that is exact at $r$. As all non-zero ranks are assigned in one of those two ways, $r$ is exact at $n-1$ inequalities other than those defining the rank of $0_{\mathcal{Z}}$, therefore, if $r \in P(\mathcal{Z}), r$ is a vertex.

Suppose that $r \notin P(\mathcal{Z})$. Then one of the cyclic flat axioms must be violated.

1. $(Z 1)$ holds by construction, as $r\left(0_{\mathcal{Z}}\right)=0$.

2. Suppose that $(Z 2)$ does not hold. By construction, $r(X)<r(Y)$ if $X \subsetneq Y$, so there exists some $X \subsetneq Y$ such that $r(Y)-r(X) \geq$ $|Y|-|X|$. As explained in Section 2.5 the inequalities arising from the cover relations imply the inequalities arising from comparable pairs more generally, so any violation of the latter implies a violation of the former. Therefore, it may be assumed that $Y$ covers $X$. Then either:

(a) $r(Y)-r(X)=1$. Therefore $|Y|-|X|=1$. Then, by Lemma 62 , there exists no matroidal rank function, and hence $P(\mathcal{Z})$ is empty.

(b) $X=0_{\mathcal{Z}}$. But, by construction,

$$
r(Y)=|Y|-1<|Y|-\left|0_{\mathcal{Z}}\right|=|Y|-0=|Y| .
$$


This is a contradiction, therefore $(Z 2)$ is not violated.

(c) $Y=1_{\mathcal{Z}}$ and $r(X)<r(Y)-1$. Let $Z \neq X$ be covered by $Y$. Then $Z$ is disjoint from $Y$, and $X \cup Z \subseteq Y$. It then follows from $r(Y)-r(X) \geq|Y|-|X| \geq|Z|$ that $r(Y) \geq|Z|+r(X)$. Therefore $r(Y)>|Z|$. However, the rank of $Y$ is given by $r(Y)=r(Z)+1$, and so then it follows from $r(Z) \leq|Z|-1$ that $r(Y) \leq|Z|$. This is a contradiction, therefore $(Z 2)$ is not violated.

3. Suppose that $(Z 3)$ does not hold. Then there exists some $X, Y \in \mathcal{Z}$ such that

$$
r(X)+r(Y)<r(X \vee Y)+r(X \wedge Y)+|X \cap Y|-|X \wedge Y| .
$$

Select a minimal such pair, i.e., a pair such that for any $Z \subsetneq X$,

$$
r(Z)+r(Y) \geq r(Z \vee Y)+r(Z \wedge Y)+|Z \cap Y|-|Z \wedge Y|
$$

and likewise for any $Z \subsetneq Y$.

If $X \subseteq Y$, axiom $(Z 2)$ is trivially satisfied, therefore, for it to be violated, $X$ and $Y$ must be incomparable. So, noting that $X \wedge Y=0_{\mathcal{Z}}$, $r\left(0_{\mathcal{Z}}\right)=0$, and $X \cap Y=\emptyset$, this reduces to $r(X)+r(Y)<r\left(1_{\mathcal{Z}}\right)$. Suppose that there exists some $Z \in \mathcal{Z}$ such that $0_{\mathcal{Z}} \subsetneq Z$ and (without loss of generality) $Z \subsetneq X$. Then $Z$ and $Y$ are incomparable. But then $Z \vee Y=1_{\mathcal{Z}}$ and $Z \wedge Y=0_{\mathcal{Z}}$, and, since, $r(Z)<r(X), r(Z)+r(Y)<$ $1_{\mathcal{Z}}$. But this contradicts the minimality of the pair $X, Y$. Therefore there is no such $Z$. Therefore $X$ and $Y$ each cover $0_{\mathcal{Z}}$. A rank function that did not violate $(Z 3)$ must either assign larger ranks to at least one of $X$ and $Y$, or a smaller rank to $1_{\mathcal{Z}}$. But $X$ and $Y$ each have the largest permitted rank, by construction, and likewise the rank of $1_{\mathcal{Z}}$ cannot be reduced without making the rank of at least one of $X$ and $Y$ smaller by the same amount, which does not alter the inequality. Therefore $P(\mathcal{Z})$ must be empty.

Therefore, if $P(\mathcal{Z})$ is nonempty, it has an integral vertex. Therefore $\mathcal{Z}$ belongs to class IV. 


\section{Chapter 4}

\section{Sums and products of lattices}

\subsection{Direct sums}

Definition 64. If $M_{1}$ and $M_{2}$ are matroids on disjoint ground sets, then the direct sum $M_{1} \oplus M_{2}$ is the matroid on $E\left(M_{1}\right) \cup E\left(M_{2}\right)$ where a set $X$ is independent in $M_{1} \oplus M_{2}$ if and only if $X=I_{1} \cup I_{2}$ for some $I_{1} \in \mathcal{I}\left(M_{1}\right)$ and $I_{2} \in \mathcal{I}\left(M_{2}\right)$.

Proposition 65. Given two matroids $M_{1}, M_{2}$ on disjoint ground sets, with cyclic flats $\mathcal{Z}\left(M_{1}\right), \mathcal{Z}\left(M_{2}\right)$, the set

$$
\left\{X_{1} \cup X_{2}: X_{1} \in \mathcal{Z}\left(M_{1}\right), X_{2} \in \mathcal{Z}\left(M_{2}\right)\right\}
$$

is the lattice of cyclic flats of the matroid $M_{1} \oplus M_{2}$, the direct sum of $M_{1}$ and $M_{2}$.

Proof. A set $F \subseteq E\left(M_{1} \oplus M_{2}\right)$ is a flat of $M_{1} \oplus M_{2}$ if and only if $F \cap E\left(M_{1}\right)$ is a flat of $M_{1}$ and $F \cap E\left(M_{2}\right)$ is a flat of $M_{2}$. Therefore $\mathcal{F}\left(M_{1} \oplus M_{2}\right)=$ $\left\{F_{1} \cup F_{2}: F_{1} \in \mathcal{F}\left(M_{1}\right), F_{2} \in \mathcal{F}\left(M_{2}\right)\right\}$. A set $C \subseteq E\left(M_{1} \oplus M_{2}\right)$ is a circuit of $M_{1} \oplus M_{2}$ if and only if it is a circuit in either $M_{1}$ or $M_{2}$. So a flat $F$ of $M_{1} \oplus M_{2}$ is cyclic if and only if $F \cap E\left(M_{1}\right)$ and $F \cap E\left(M_{2}\right)$ are both cyclic. Therefore $F \in \mathcal{Z}\left(M_{1} \oplus M_{2}\right)$ is equivalent to both of $F \cap E\left(M_{1}\right) \in \mathcal{Z}\left(M_{1}\right)$ and $F \cap E\left(M_{2}\right) \in \mathcal{Z}\left(M_{2}\right)$ being simultaneously true. 


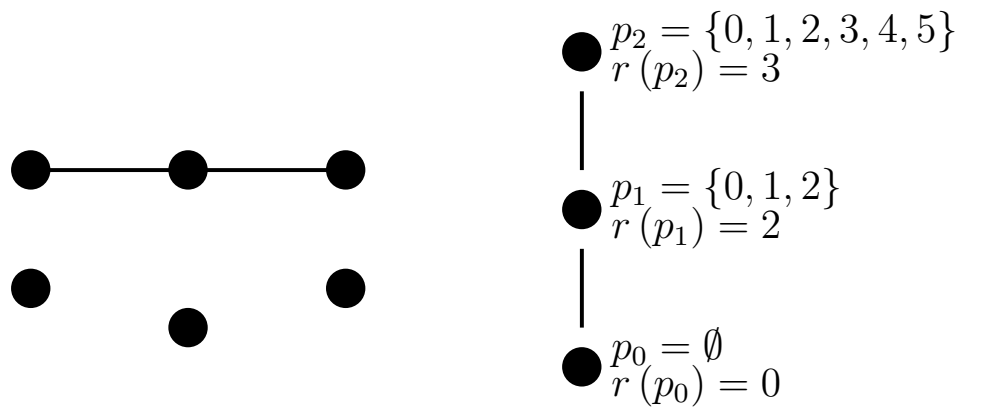

Figure 4.1.1: The matroid $P_{6}$ and its cyclic flats

The direct sum operation on matroids then gives rise to an operation on lattices of sets, that we shall refer to using the same name and notation.

Definition 66 . If $\mathcal{Z}_{1}$ and $\mathcal{Z}_{2}$ are lattices of sets on disjoint ground sets, their direct $\operatorname{sum} \mathcal{Z}_{1} \oplus \mathcal{Z}_{2}$ is the set

$$
\mathcal{Z}_{1} \oplus \mathcal{Z}_{2}=\left\{Z_{1} \cup Z_{2}: Z_{1} \in \mathcal{Z}_{1}, Z_{2} \in \mathcal{Z}_{2}\right\}
$$

There is an obvious lattice isomorphism between $\mathcal{Z}_{1} \oplus \mathcal{Z}_{2}$ and the Cartesian product $\mathcal{Z}_{1} \times \mathcal{Z}_{2}$, but in the context of matroids it is the elements of the sets that are important, and not merely the lattice structure. There is also an unfortunate overloading of the $\oplus$ operator, which is used for the linear sum of posets. The $\oplus$ symbol shall only be used in this thesis for the direct sum operation defined here.

Lemma 67. If $\mathcal{Y}$ and $\mathcal{Z}$ are matroidal lattices, then $\mathcal{Y} \oplus \mathcal{Z}$ is a matroidal lattice.

Proof. This follows directly from the fact that, if $M_{1}$ and $M_{1}$ are matroids, $\mathcal{Z}\left(M_{1} \oplus M_{2}\right)=\mathcal{Z}\left(M_{1}\right) \oplus \mathcal{Z}\left(M_{2}\right)$.

This means that class $\mathrm{V}$ is closed under the direct sum operation.

As we believe that all lattices belong to class IV, we therefore also expect class IV to be closed under the direct sum operation.

Lemma 68. Classes I, II, and III are not closed under the direct sum operation. 


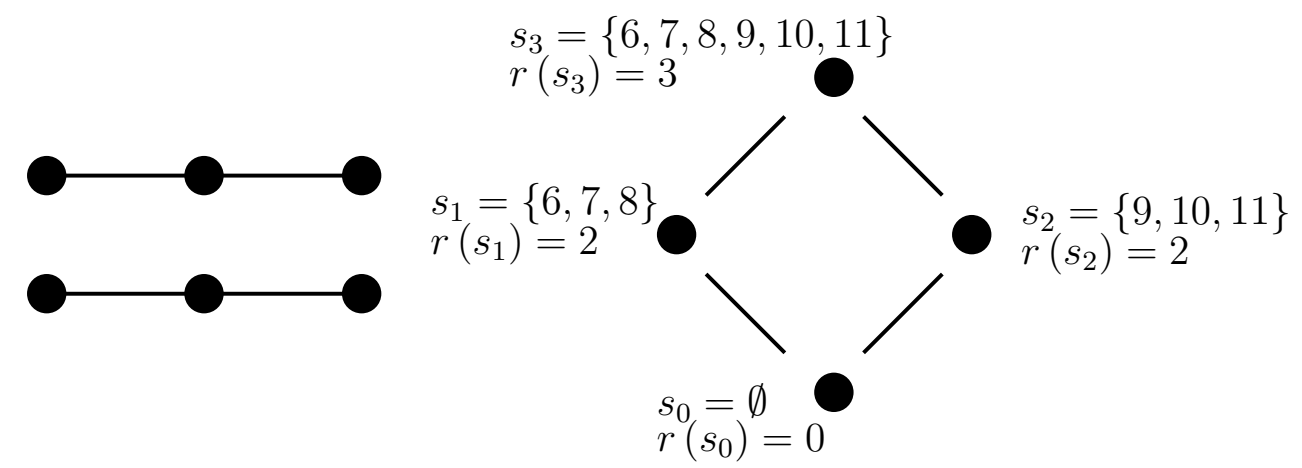

Figure 4.1.2: The matroid $R_{6}$ and its cyclic flats

Proof. The $P_{6}$ matroid, shown in Figure 4.1 .1 on page 38, is a matroid on 6 elements. The lattice of cyclic flats of $P_{6}$ is a single chain (Figure 4.1.1), and so the constraint matrix is totally unimodular by Lemma 61 .

The $R_{6}$ matroid, shown in Figure 4.1.2, is a matroid on 6 elements. The lattice of cyclic flats of $R_{6}$ has width two and height three, and therefore the constraint matrix of its lattice of cyclic flats is also totally unimodular, as will be shown in Chapter 6 .

As both lattices are those of actual matroids, their polyhedra are clearly nonempty. Further, as the matrices are totally unimodular, the lattices belong to class I (and therefore also classes II and III). However, their direct sum, $\mathcal{Z}_{P_{6}} \oplus \mathcal{Z}_{R_{6}}$, has a polyhedron with half-integral vertices. For instance, the point

$$
\mathrm{x}=\left(0,2, \frac{7}{2}, 2, \frac{7}{2}, 5,2, \frac{7}{2}, 5,4,5,6\right)
$$

is a half-integral vertex. To see this, first look at the lattice of cyclic flats (Figure 4.1 .3 on page 41).

A linearly independent set of inequalities on the ranks of these sets, derived from the cyclic flat inequalities, is: 


$$
\begin{array}{rrr}
r\left(x_{1}\right)-r\left(x_{0}\right) \leq 2 & =2-0 \\
r\left(x_{3}\right)-r\left(x_{0}\right) \leq 2 & =2-0 \\
r\left(x_{6}\right)-r\left(x_{0}\right) \leq 2 & =6-0 \\
r\left(x_{10}\right)-r\left(x_{9}\right) \geq 1 & =5-4 \\
r\left(x_{11}\right)-r\left(x_{5}\right) \geq 1 & =6-5 \\
r\left(x_{11}\right)-r\left(x_{8}\right) \geq 1 & =6-5 \\
r\left(x_{11}\right)-r\left(x_{10}\right) \geq 1 & =6-5 \\
r\left(x_{2}\right)+r\left(x_{4}\right)-r\left(x_{1}\right)-r\left(x_{5}\right) \geq 0 & =\frac{7}{2}+\frac{7}{2}-2-5 \\
r\left(x_{2}\right)+r\left(x_{7}\right)-r\left(x_{1}\right)-r\left(x_{8}\right) \geq 0 & =\frac{7}{2}+\frac{7}{2}-2-5 \\
r\left(x_{3}\right)+r\left(x_{6}\right)-r\left(x_{0}\right)-r\left(x_{9}\right) \geq 0 & =2+2-0-4 \\
r\left(x_{4}\right)+r\left(x_{7}\right)-r\left(x_{1}\right)-r\left(x_{10}\right) \geq 0 & =\frac{7}{2}+\frac{7}{2}-2-5
\end{array}
$$

As can be seen by the substitutions on the right-hand side above, these inequalities are exact at the point $\mathrm{x}$. To demonstrate that this is a feasible point is a trivial exercise and, therefore, this shows that $\mathrm{x}$ is a vertex, despite not being integral. Therefore, the lattice $\mathcal{Z}_{P_{6}} \oplus \mathcal{Z}_{R_{6}}$ is not a class-III lattice. Therefore, none of classes I, II, and III are closed under direct sums.

\subsection{Free products}

The free product $M_{1} \square M_{2}$ of two matroids on disjoint ground sets is a noncommutative operation defined by Crapo and Schmitt in 2005 [2]. Bonin and de Mier showed that the cyclic flats provided a conceptually simple way of understanding the free product [1]. We use their definition here.

Definition 69. If $M_{1}$ and $M_{2}$ are matroids on disjoint ground sets, then the free product $M_{1} \square M_{2}$ is the matroid that has ground set $E\left(M_{1}\right) \cup E\left(M_{2}\right)$ 


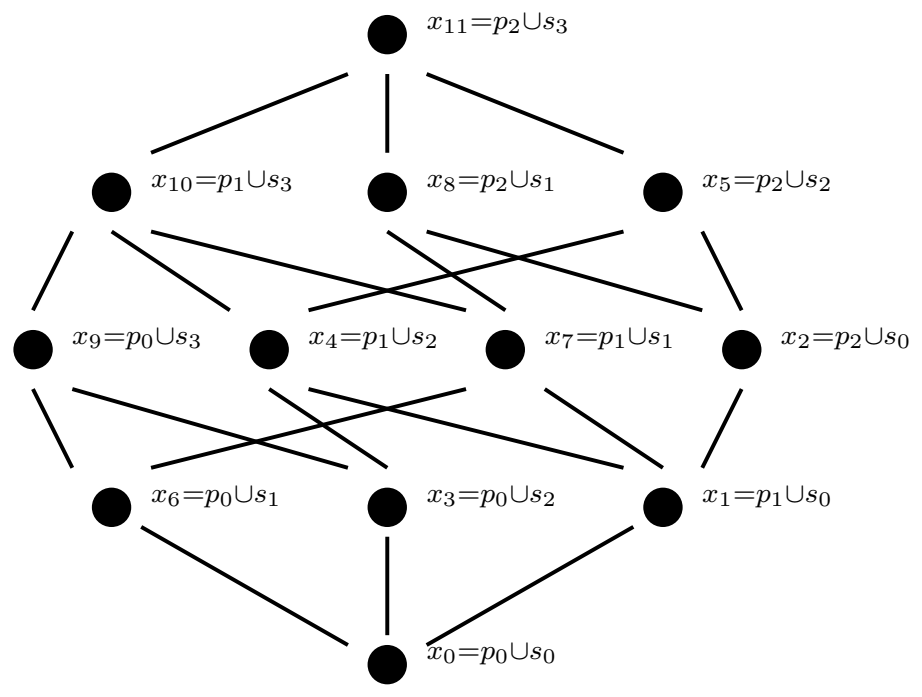

Figure 4.1.3: Lattice of cyclic flats of $R_{6} \oplus P_{6}$

with cyclic flats

$$
\mathcal{Z}\left(M_{1} \square M_{2}\right)=\left(\mathcal{Z}\left(M_{1}\right) \backslash\left\{E\left(M_{1}\right)\right\}\right) \cup \mathcal{Z}^{\dagger}\left(M_{2}\right) \cup Q
$$

where

$$
\mathcal{Z}^{\dagger}\left(M_{2}\right)=\left\{Z \cup E\left(M_{1}\right): Z \in \mathcal{Z}\left(M_{2}\right)\right\}
$$

and

$$
Q= \begin{cases}\left\{E\left(M_{1}\right)\right\}, & M_{1} \text { has no coloops and } M_{2} \text { has no loops } \\ \emptyset, & \text { otherwise }\end{cases}
$$

and where the ranks of those cyclic flats are given by $r_{M_{1} \square M_{2}}(X)=r_{M_{1}}(X)$ if $X \subseteq E\left(M_{1}\right)$, and $r_{M_{1} \square M_{2}}\left(E\left(M_{1}\right) \cup Y\right)=r_{M_{1}}\left(E\left(M_{1}\right)\right)+r_{M_{2}}(Y)$ otherwise.

We use this as a basis for a definition of the free product operation on lattices of sets.

Definition 70 . If $\mathcal{Z}_{1}$ and $\mathcal{Z}_{2}$ are lattices of sets on disjoint ground sets, then the free product $\mathcal{Z}_{1} \square \mathcal{Z}_{2}$ is the lattice given by

$$
\mathcal{Z}_{1} \square \mathcal{Z}_{2}=\left\{X: X \in \mathcal{Z}_{1}, X \neq E\left(\mathcal{Z}_{1}\right)\right\} \cup\left\{E\left(\mathcal{Z}_{1}\right) \cup Y: Y \in \mathcal{Z}_{2}, Y \neq \emptyset\right\} \cup Q
$$


where

$$
Q= \begin{cases}\left\{E\left(\mathcal{Z}_{1}\right)\right\}, & 1_{\mathcal{Z}\left(M_{1}\right)}=E\left(M_{1}\right) \text { and } 0_{\mathcal{Z}\left(M_{2}\right)}=\emptyset \\ \emptyset, & \text { otherwise. }\end{cases}
$$

It can easily be seen that the lattice structure arising from this operation is isomorphic to either the linear sum of the two separate lattices, or the lattice arising from the identification of the greatest element of $\mathcal{Z}_{1}$ and the least element of $\mathcal{Z}_{2}$, depending on whether $\mathcal{Z}_{1}$ contains coloops and $\mathcal{Z}_{2}$ contains loops.

Proposition 71 . If $\mathcal{Y}$ and $\mathcal{Z}$ are matroidal lattices, then $\mathcal{Y} \square \mathcal{Z}$ is a matroidal lattice.

Proof. This follows directly from the fact that $\mathcal{Z}\left(M_{1} \square M_{2}\right)=\mathcal{Z}\left(M_{1}\right)$ $\mathcal{Z}\left(M_{2}\right)$.

This means that class $\mathrm{V}$ is closed under the free product operation.

Theorem 72. Let $\mathcal{Y}, \mathcal{Z}$ be class-I lattices of sets. Then $\mathcal{Y} \square \mathcal{Z}$ is a class-I lattice.

Proof. Let the constraint matrices for $\mathcal{Y}$ and $\mathcal{Z}$ have the forms

$$
\begin{gathered}
C(\mathcal{Y})=\left[P \mid 1_{\mathcal{Z}}\right] \\
C(\mathcal{Z})\left[\begin{array}{c|c}
1 & 0 \\
-1 & 0 \\
0_{\mathcal{Z}}^{\prime} & Q
\end{array}\right]
\end{gathered}
$$

where $1_{\mathcal{Y}}$ refers to the rightmost column of $C(\mathcal{Y})$ and $0_{\mathcal{Z}}^{\prime}$ to the leftmost column of $C(\mathcal{Z})$, excluding the first two rows (those that set the rank of the $0_{\mathcal{Z}}$ element).

Construct the constraint matrix for $\mathcal{Y} \square \mathcal{Z}$. It can have one of two possible forms, depending on whether $\mathcal{Y}$ contains coloops and whether $\mathcal{Z}$ contains loops.

In the first form, it will have the structure 


$$
C(\mathcal{Y} \square \mathcal{Z})=\left(\begin{array}{c|cc|c}
P & 1 \mathcal{Y} & 0 & 0 \\
0 & 1 & -1 & 0 \\
0 & -1 & 1 & 0 \\
0 & 0 & 0_{\mathcal{Z}}^{\prime} & Q
\end{array}\right)
$$

So take some subset $X$ of columns of $C(\mathcal{Y} \square \mathcal{Z})$. There is a clear correspondence between columns of $C(\mathcal{Y} \square \mathcal{Z})$ and columns of $C(\mathcal{Y})$ and $C(\mathcal{Z})$, where a column of $C(\mathcal{Y} \square \mathcal{Z})$ is simply either a column of $C(\mathcal{Y})$ with additional elements appended, or a column of $C(\mathcal{Z})$ with additional elements prepended. Then say that $X=Y \cup Z$, where $Y$ is a subset of columns of $C(\mathcal{Y} \square \mathcal{Z})$ that have this correspondence with some subset $Y^{\prime}$ of columns of $C(\mathcal{Y})$, and $Z$ is a subset of columns of $C(\mathcal{Y} \square \mathcal{Z})$ that have this correspondence with some subset $Z^{\prime}$ of $C(\mathcal{Z})$. Examining the columns of $C(\mathcal{Y} \square \mathcal{Z})$, it is clear that Proposition 44 applies equally well to $Y$ and to $Z$ as it does to $Y^{\prime}$ and $Z^{\prime}$, and so let $\left(\epsilon_{j}\right)_{j \leq|Y|},\left(\zeta_{k}\right)_{k \leq|Z|}$ be appropriate sequences such that $\epsilon_{j}= \pm 1, \zeta_{k}= \pm 1$, and $\sum \epsilon_{i} Y_{i}$ and $\sum \zeta_{i} Z_{i}$ are both totally unimodular vectors. Clearly, if $\sum \zeta_{i} Z_{i}$ is totally unimodular, then $-\sum \zeta_{i} Z_{i}$ is as well, therefore $\left(-\zeta_{k}\right)$ is also an appropriate sequence for Proposition 44 . So, if the central two columns of $C(\mathcal{Y} \square \mathcal{Z})$ are both in $X$ and have indices $m$ and $n$ in $Y$ and $Z$, respectively, choose a sequence $\left(\zeta_{k}\right)$ such that $\epsilon_{m}=\zeta_{n}$. Then, by examination of the structure of $C(\mathcal{Y} \square \mathcal{Z})$, it is clear that $\sum \epsilon_{i} Y_{i}+\sum \zeta_{i} Z_{i}$ is a totally unimodular vector, and so there exists a sequence $\left(\eta_{l}\right)_{l \leq|X|}$ such that $\eta_{l}= \pm 1$ and $\sum \eta_{i} X_{i}$ is a totally unimodular vector. Therefore, by Proposition 44, $C(\mathcal{Y} \square \mathcal{Z})$ is totally unimodular.

In the second possible form, the constraint matrix of $\mathcal{Y} \square \mathcal{Z}$ will have the structure

$$
C(\mathcal{Y} \square \mathcal{Z})=\left(\begin{array}{c|c|c}
P & 1_{\mathcal{Y}} & 0 \\
0 & 0_{\mathcal{Z}}^{\prime} & Q
\end{array}\right)
$$

where the $1_{\mathcal{Y}}$ and $0_{\mathcal{Z}}$ are mapped to the same column in $C(\mathcal{Y} \square \mathcal{Z})$.

Then given a subset $X$ of columns of $C(\mathcal{Y} \square \mathcal{Z})$, take $Y$ and $Z$ where $X=Y \cup Z$, in the same manner as in the previous case, except that now 
$Y$ and $Z$ may share a single element (the central column of $C(\mathcal{Y} \square \mathcal{Z})$ is both a column of $C(\mathcal{Y})$ with elements appended and a column of $C(\mathcal{Z})$ with elements prepended). Say that the labels $1_{\mathcal{Y}}$ and $0_{\mathcal{Z}}$ both refer to the central column of $C(\mathcal{Y} \square \mathcal{Z})$. So $Y$ and $Z$ are disjoint unless $1_{\mathcal{Y}}=0_{\mathcal{Z}} \in X$, in which case $Y \cap Z=\left\{1_{\mathcal{Y}}\right\}$. Choose sequences $\left(\epsilon_{j}\right)_{j \leq|Y|},\left(\zeta_{k}\right)_{k \leq|Z|}$ in accordance with Proposition 44 such that, if $1_{\mathcal{Y}}=0_{\mathcal{Z}} \in X$, then that column has index $m$ in both $Y$ and $Z$, and $\epsilon_{m}=\zeta_{m}$. Then construct the sequence $\left(\eta_{l}\right)_{l \leq|X|}$ by letting $\eta_{j}=\epsilon_{j}$ for all $1 \leq j \leq|Y|$ and $\eta_{k+|Y|}=\zeta_{k}$ for all $1 \leq k \leq|Z|$ where $k \neq m$, and assign indices to the elements of $X$ such that $X_{j}=Y_{j}$ for all $j$, and $X_{|Y|+k}=Z_{k}$ for all $k \neq m$. Again, by inspection of the structure of $C(\mathcal{Y} \square \mathcal{Z})$, it is easily seen that $\sum \eta_{i} X_{i}$ is totally unimodular. Hence, again by Proposition $44, \mathcal{Y} \square \mathcal{Z}$ has a totally unimodular constraint matrix.

Theorem 73. Let $\mathcal{Y}, \mathcal{Z}$ be class-II lattices of sets (i.e., have unimodular constraint matrices). Then $\mathcal{Y} \square \mathcal{Z}$ is a class-II lattice.

Proof. By Proposition 47, the transpose of the constraint matrix $\mathcal{C}(\mathcal{Y} \square \mathcal{Z})^{T}$ is unimodular if and only if there exists a unimodular basis $B$ of $\mathcal{C}(\mathcal{Y} \square \mathcal{Z})^{T}$ such that the unique matrix $D$ satisfying $B D=\mathcal{C}(\mathcal{Y} \square \mathcal{Z})^{T}$ is totally unimodular.

As in Theorem 72, $A$ can have one of two forms. In the first form

$$
C(\mathcal{Y} \square \mathcal{Z})=\left(\begin{array}{c|cc|c}
P & 1 \mathcal{Y} & 0 & 0 \\
0 & 1 & -1 & 0 \\
0 & -1 & 1 & 0 \\
0 & 0 & 0_{\mathcal{Z}}^{\prime} & Q
\end{array}\right)
$$

As $C(\mathcal{Y})$ and $C(\mathcal{Z})$ are unimodular, there exist unimodular bases $B_{\mathcal{Y}}, B_{\mathcal{Z}}$ of the transpose of each such that the matrices $D_{\mathcal{Y}}, D_{\mathcal{Z}}$ satisfying $B_{\mathcal{Y}} D_{\mathcal{Y}}=$ $C(\mathcal{Y})^{T}$ and $B_{\mathcal{Z}} D_{\mathcal{Z}}=C(\mathcal{Z})^{T}$ are totally unimodular. The rows defining the rank of the zero element of $\mathcal{Z}$ are not present in $C(\mathcal{Y} \square \mathcal{Z})$, and, as shown in Lemma 52, one of these rows (which are columns in the transpose matrix) must be present in every basis of $C(\mathcal{Z})^{T}$. Let $B_{\mathcal{Z}}^{\prime}$ be $B_{\mathcal{Z}}$ with that row (column) removed. By inspection, it can be seen that the rows (columns) of 
$B_{\mathcal{Y}}$ and $B_{\mathcal{Z}}^{\prime}$, plus one of the central two rows (columns) of $C(\mathcal{Y} \square \mathcal{Z})$ make up a basis of $C(\mathcal{Y} \square \mathcal{Z})^{T}$. So choose that central row which has the same sign in the $0_{\mathcal{Z}}^{\prime}$ column as the row removed from $B_{\mathcal{Z}}$ has. Call this row (column) a. Then $B=B_{\mathcal{Y}} \cup B_{\mathcal{Z}}^{\prime} \cup\{a\}$ is a basis of $C(\mathcal{Y} \square \mathcal{Z})^{T}$.

As $B$ is a basis and therefore nonsingular, to show that $B$ is unimodular, it suffices to show that $|B|= \pm 1$. There are two elementary column operations that change the sign of the determinant of a matrix, but not its magnitude: interchanging two columns, and adding a multiple of one column to another. So then $B$ is unimodular if and only if the identity matrix can be obtained solely through these operations.

As $B_{\mathcal{Y}}$ is unimodular, the identity matrix $I_{|\mathcal{Y}|}$ can be obtained in the top left corner of $B$ through these elementary column operations. Then it is easy to see that adding a multiple of the $|\mathcal{Y}|$ th column to the $|\mathcal{Y}|+1$ th column will give the matrix

$$
\left(\begin{array}{cc}
I_{|\mathcal{Y}|} & 0 \\
0 & B_{\mathcal{Z}}
\end{array}\right)
$$

and it is clear that, since $B_{\mathcal{Z}}$ is unimodular, the specified elementary column operations will allow this matrix to be transformed into the identity matrix. Therefore $B$ is unimodular.

The matrix given by

$$
D^{T}=\left(\begin{array}{cc}
{\left[D_{\mathcal{Y}}\right]^{T}} & 0 \\
0 & {\left[D_{\mathcal{Z}}\right]^{T}}
\end{array}\right)
$$

constructed in the same way as the $C(\mathcal{Y} \square \mathcal{Z})$, as an overlapping block matrix, satisfies $B D=C(\mathcal{Y} \square \mathcal{Z})$. Furthermore, by Theorem 72 , it is totally unimodular.

So there exists a unimodular basis $B$ of $C(\mathcal{Y} \square \mathcal{Z})^{T}$ such that the unique matrix $D$ satisfying $B D=C(\mathcal{Y} \square \mathcal{Z})$ is totally unimodular. Therefore $C(\mathcal{Y} \square \mathcal{Z})$ is a unimodular matrix.

Then suppose that the matrix $C(\mathcal{Y} \square \mathcal{Z})$ has the second form. 


$$
C(\mathcal{Y} \square \mathcal{Z})=\left(\begin{array}{c|c|c}
P & 1_{\mathcal{Y}} & 0 \\
0 & 0_{\mathcal{Z}}^{\prime} & Q
\end{array}\right)
$$

Then, by similar reasoning as used above, it can be seen that $B=B_{\mathcal{Y}} \cup B_{\mathcal{Z}}^{\prime}$ is a basis for $C(\mathcal{Y} \square \mathcal{Z})^{T}$. Following the same steps as above, using the specified elementary column operations to reduce the matrix, it is possible to obtain

$$
\left(\begin{array}{cc}
I_{|\mathcal{Y}|} & 0 \\
0 & B_{\mathcal{Z}}^{\prime}
\end{array}\right)
$$

and by multiplication of the $|\mathcal{Y}|$ th column by \pm 1 , this can be transformed into

$$
\left(\begin{array}{cc}
I_{|\mathcal{Y}|-1} & 0 \\
0 & B_{\mathcal{Z}}
\end{array}\right)
$$

which can then clearly be reduced to the identity matrix. Therefore $B$ is unimodular.

Letting $D_{\mathcal{Z}}^{\prime}$ by the matrix obtained by removing the initial row from $D_{\mathcal{Z}}$, then the matrix given by

$$
D^{T}=\left(\begin{array}{cc}
{\left[D_{\mathcal{Y}}\right]^{T}} & 0 \\
0 & {\left[D_{\mathcal{Z}}^{\prime}\right]^{T}}
\end{array}\right)
$$

satisfies $B D=C(\mathcal{Z})$, and, since the total unimodularity of $D_{\mathcal{Z}}$ implies the total unimodularity of $D_{\mathcal{Z}}^{\prime}$, it again is totally unimodular. Therefore, by similar reasoning as in the first case above, $C(\mathcal{Y} \square \mathcal{Z})$ is totally unimodular.

Therefore $\mathcal{Y} \square \mathcal{Z}$ is a class II lattice.

It is not known whether class III is closed under the free product operation.

Problem 74. If $\mathcal{Y}$ and $\mathcal{Z}$ are lattices belong to class III, does $\mathcal{Y} \square \mathcal{Z}$ belong to class III?

Although it is clear that, for vertices $v$ and $u$ of $P(Y)$ and $P(Z)$, respectively, the concatenation of $v$ and $u+x$ (where $x$ is an integer, the value 
of which depends on the nature of the lattice $\mathcal{Y} \square \mathcal{Z}$ ) will be a vertex of $P(\mathcal{Y} \square \mathcal{Z})$, there may be additional vertices of $P(\mathcal{Y} \square \mathcal{Z})$ that are not of this form.

The belief that all lattices belong to class IV (Conjecture 29) implies the following.

Conjecture 75. Class IV is closed under the free product operation. 


\section{Chapter 5}

\section{Duals}

\subsection{Lattice duals}

Definition 76. The order dual of a partial order $\mathbb{P}=(P, \leq)$ is an order $\mathbb{P}^{*}=\left(P, \leq_{*}\right)$, where $a \leq_{*} b$ in $\mathbb{P}^{*}$ if and only if $b \leq a$ in $\mathbb{P}$.

Lemma 77. Let $M$ be a matroid with ground set $E$. A flat $F$ of $M$ is cyclic if and only if the complement of $F$ is a coflat of $M$.

Proof. Let $F$ be a flat of $M$, and $E$ the ground set of $M$. If $e \in F$, then $e$ is contained in a circuit in $F$ if and only if $e$ is not in a cocircuit contained in $(E-F) \cup\{e\}$. Therefore $e$ is in a circuit in $F$ if and only if $F$ is disjoint from the coclosure of $E-F$.

Corollary 78. A set $Z \subseteq E$ is a cyclic flat of $M$ if and only if $E \backslash Z$ is a cyclic flat of the dual matroid $M^{*}$.

Proof. This follows directly from Lemma 77.

Corollary 79. If $\mathcal{Z}$ is a lattice of subsets of a ground set $E$, then $\mathcal{Z}^{*}=$ $\{E \backslash Z: Z \in \mathcal{Z}\}$ is a lattice of subsets of $E$.

Proof. If $X \in \mathcal{Z}$, then $\bar{X} \in \mathcal{Z}^{*}$, where $\bar{X}$ is the complement of $X$ in $E$. Clearly, if $X \subseteq Y$ for some $X, Y \in \mathcal{Z}$, then $\bar{Y} \subseteq \bar{X}$. It is obvious that $\mathcal{Z}^{*}$ 
is isomorphic to the order dual of $\mathcal{Z}$, and the order dual of a lattice is a lattice.

We call $\mathcal{Z}^{*}$ the dual lattice of $\mathcal{Z}$, by analogy with the dual operation on matroids. The lattice structure of $\mathcal{Z}^{*}$ is simply that of $\mathcal{Z}$, flipped upside down.

Lemma 80. Classes I and II are closed under duality.

Proof. The constraint matrix depends entirely on the lattice structure. If $Y$ covers $X$ in $\mathcal{Z}$, then $\bar{X}$ covers $\bar{Y}$ in $\mathcal{Z}^{*}$, and therefore the rows

$$
\begin{aligned}
& \begin{array}{lllllll}
0_{\mathcal{Z}} & \cdots & X & \cdots & Y & \cdots & 1_{\mathcal{Z}}
\end{array} \\
& \left(\begin{array}{ccccccc}
0 & \cdots & 1 & \cdots & -1 & \cdots & 0 \\
0 & \cdots & -1 & \cdots & 1 & \cdots & 0
\end{array}\right)
\end{aligned}
$$

would be replaced by

$$
\begin{aligned}
& \begin{array}{lllllll}
\overline{0}_{\mathcal{Z}} & \ldots & \bar{X} & \ldots & \bar{Y} & \ldots & \overline{1}_{\mathcal{Z}}
\end{array} \\
& \left(\begin{array}{ccccccc}
0 & \cdots & -1 & \cdots & 1 & \cdots & 0 \\
0 & \cdots & 1 & \cdots & -1 & \cdots & 0
\end{array}\right)
\end{aligned}
$$

while for an incomparable pair $X, Y \in \mathcal{Z}, \bar{X}$ and $\bar{Y}$ are also incomparable, and so the row

$$
\left.\begin{array}{ccccccccccc}
0_{\mathcal{Z}} & \cdots & X \wedge Y & \cdots & X & \cdots & Y & \cdots & X \vee Y & \cdots & 1_{\mathcal{Z}} \\
0 & \cdots & 1 & \cdots & -1 & \cdots & -1 & \cdots & 1 & \cdots & 0
\end{array}\right)
$$

would be replaced by

$$
\left.\begin{array}{ccccccccccc}
\overline{0}_{\mathcal{Z}} & \ldots & \overline{X \wedge Y} & \ldots & \bar{X} & \ldots & \bar{Y} & \ldots & \overline{X \vee Y} & \ldots & \overline{1}_{\mathcal{Z}} \\
0 & \ldots & 1 & \ldots & -1 & \ldots & -1 & \ldots & 1 & \ldots & 0
\end{array}\right)
$$

Both of these replacements clearly do not alter the constraint matrix in a significant way. The only meaningful difference, then, between $C(\mathcal{Z})$ and $C\left(\mathcal{Z}^{*}\right)$ is that the rows

$$
\begin{aligned}
& \begin{array}{lll}
0_{\mathcal{Z}} & \cdots & 1_{\mathcal{Z}}
\end{array} \\
& \left(\begin{array}{ccc}
1 & \cdots & 0 \\
-1 & \cdots & 0
\end{array}\right)
\end{aligned}
$$


are replaced by

$$
\begin{gathered}
\overline{0}_{\mathcal{Z}} \\
\left(\begin{array}{ccc}
0 & \cdots & \overline{1}_{\mathcal{Z}} \\
0 & \cdots & 1 \\
-1
\end{array}\right)
\end{gathered}
$$

Total unimodularity and unimodularity are both preserved by adding (or removing) rows with at most one nonzero entry, that entry being 1 or -1 . Therefore $C\left(\mathcal{Z}^{*}\right)$ must be unimodular if $C(\mathcal{Z})$ is unimodular, and totally unimodular if $C(\mathcal{Z})$ is totally unimodular.

\subsection{Polyhedra of dual lattices}

Theorem 81. Let $\mathcal{Z}$ be a lattice of sets on ground set $E$, and $P(\mathcal{Z})$ the polyhedron generated from it. Let $r \in P(\mathcal{Z})$. Then $r^{*} \in P\left(\mathcal{Z}^{*}\right)$, where $r^{*}$ is defined by

$$
r^{*}(X)=|X|-r(M)+r(\bar{X})
$$

for all $X \in \mathcal{Z}^{*}$, and

$$
r(M)=r\left(1_{\mathcal{Z}}\right)+\left|E \backslash 1_{\mathcal{Z}}\right| .
$$

Proof. First, we show that $r^{*}\left(0_{\mathcal{Z}^{*}}\right)=0$. The complement of $0_{\mathcal{Z}^{*}}$ is $1_{\mathcal{Z}}$, and $\left|0_{\mathcal{Z}^{*}}\right|=\left|E \backslash 1_{\mathcal{Z}}\right|$. Therefore

$$
r^{*}\left(0_{\mathcal{Z}^{*}}\right)=\left|E \backslash 1_{\mathcal{Z}}\right|-\left(r\left(1_{\mathcal{Z}}\right)+\left|E \backslash 1_{\mathcal{Z}}\right|\right)+r\left(1_{\mathcal{Z}}\right)=0 .
$$

Suppose that $X \subsetneq Y$ for some $X, Y \in \mathcal{Z}^{*}$. Note then that $\bar{Y} \subsetneq \bar{X}$. Then

$$
r(\bar{X})-r(\bar{Y}) \leq|\bar{X}|-|\bar{Y}|-1,
$$

which can be rearranged to give

$$
r(\bar{X})-|\bar{X}|+1 \leq r(\bar{Y})-|\bar{Y}|
$$

and, through the addition of $|E|-r(M)$, it can be seen that

$$
|E|-|\bar{X}|-r(M)+r(\bar{X})+1 \leq|E|-|\bar{Y}|-r(M)+r(\bar{Y}),
$$


meaning that

$$
|X|-r(M)+r(\bar{X})+1 \leq|Y|-r(M)+r(\bar{Y})
$$

and therefore

$$
r^{*}(X)+1 \leq r^{*}(Y)
$$

By a similar process, it is easy to see that

$$
\begin{aligned}
& r(\bar{Y})+1 \leq r(\bar{X}) \\
& \Rightarrow|Y|-r(M)+r(\bar{Y})-|Y|+1 \leq|X|-r(M)+r(\bar{X})-|X| \\
& \Rightarrow \quad r^{*}(Y)-|Y|+1 \leq r^{*}(X)-|X| \\
& \Rightarrow \quad r^{*}(Y)-r^{*}(X) \leq|Y|-|X|-1 \text {. }
\end{aligned}
$$

Therefore $r^{*}$ complies with $(Z 2)$.

Finally, for some $X, Y \in \mathcal{Z}^{*}$ (and complementary $\bar{X}, \bar{Y} \in \mathcal{Z}$ ), using $\wedge_{*}$ and $\vee_{*}$ to refer to the meet and join operations on $\mathcal{Z}^{*}$,

$$
r(\bar{X})+r(\bar{Y}) \geq r(\bar{X} \vee \bar{Y})+r(\bar{X} \wedge \bar{Y})+|\bar{X} \cap \bar{Y}|-|\bar{X} \wedge \bar{Y}|,
$$

which, using the facts that $|\bar{X} \cap \bar{Y}|=|\overline{X \cup Y}|$ and $|\bar{X} \wedge \bar{Y}|=\left|\overline{X \vee_{*} Y}\right|$, gives

$$
r(\bar{X})+r(\bar{Y}) \geq r(\bar{X} \vee \bar{Y})+r(\bar{X} \wedge \bar{Y})+\left|X \vee_{*} Y\right|-|X \cup Y|
$$

Then, as $|X \cup Y|=|X|+|Y|-|X \cap Y|$,

$r(\bar{X})+r(\bar{Y})+|X|+|Y| \geq r(\bar{X} \vee \bar{Y})+r(\bar{X} \wedge \bar{Y})+\left|X \vee_{*} Y\right|+|X \cap Y|$

giving

$r(\bar{X})+r(\bar{Y})+|X|+|Y| \geq r\left(\overline{X \wedge_{*} Y}\right)+r\left(\overline{X \vee_{*} Y}\right)+\left|X \vee_{*} Y\right|+|X \cap Y|$,

meaning that

$$
\begin{aligned}
& |X|+r(\bar{X})+|Y|+r(\bar{Y}) \geq \\
& \left|X \vee_{*} Y\right|+r\left(\overline{X \vee_{*} Y}\right)+\left|X \wedge_{*} Y\right|+r\left(\overline{X \wedge_{*} Y}\right)+|X \cap Y|-\left|X \wedge_{*} Y\right|
\end{aligned}
$$


and therefore

$$
r^{*}(X)+r^{*}(Y) \geq r^{*}\left(X \vee_{*} Y\right)+r^{*}\left(X \wedge_{*} Y\right)+|X \cap Y|-\left|X \wedge_{*} Y\right|
$$

Therefore $r^{*}$ complies with $(Z 3)$. Therefore $r^{*} \in P\left(\mathcal{Z}^{*}\right)$.

Lemma 82. The operation $r \mapsto r^{*}$ is an involution between $P(\mathcal{Z})$ and $P\left(\mathcal{Z}^{*}\right)$.

Proof. Given $r \in P(\mathcal{Z})$, consider $r^{* *}$. For some $X \in \mathcal{Z}^{*}$, and complementary $\bar{X} \in \mathcal{Z}$, we first find an expression for $r^{*}\left(1_{\mathcal{Z}}\right)$. By definition, it is

$$
r^{*}\left(1_{\mathcal{Z}^{*}}\right)=\left|1_{\mathcal{Z}^{*}}\right|-r(M)+r\left(E \backslash 1_{\mathcal{Z}^{*}}\right)
$$

which, through the use of obvious substitions, gives

$$
r^{*}\left(1_{\mathcal{Z}^{*}}\right)=\left|E \backslash 0_{\mathcal{Z}}\right|-r\left(1_{\mathcal{Z}}\right)-\left|E \backslash 1_{\mathcal{Z}}\right|+r\left(0_{\mathcal{Z}}\right)
$$

which can then be simplified to

$$
r^{*}\left(1_{\mathcal{Z}^{*}}\right)=\left|1_{\mathcal{Z}}\right|-\left|0_{\mathcal{Z}}\right|-r\left(1_{\mathcal{Z}}\right) .
$$

Since

$$
r^{*}(X)=|X|-r(M)+r(\bar{X})
$$

and $r(M)=r\left(1_{\mathcal{Z}}\right)+\left|E \backslash 1_{\mathcal{Z}}\right|$, then

$$
r^{*}(X)=|X|-r\left(1_{\mathcal{Z}}\right)-\left|E \backslash 1_{\mathcal{Z}}\right|+r(\bar{X}) .
$$

Finally, we take the definition for $r^{* *}(\bar{X})$,

$$
r^{* *}(\bar{X})=|\bar{X}|-r^{*}\left(M^{*}\right)+r^{*}(X),
$$

replace $r^{*}\left(M^{*}\right)$ with $r^{*}\left(1_{\mathcal{Z}^{*}}\right)+\left|E \backslash 1_{\mathcal{Z}^{*}}\right|$,

$$
r^{* *}(\bar{X})=|\bar{X}|-r^{*}\left(1_{\mathcal{Z}^{*}}\right)-\left|E \backslash 1_{\mathcal{Z}^{*}}\right|+r^{*}(X),
$$

and substitute out $r^{*}\left(1_{\mathcal{Z}^{*}}\right)$ for the expression obtained earlier to give $r^{* *}(\bar{X})=|\bar{X}|-\left|1_{\mathcal{Z}}\right|+\left|0_{\mathcal{Z}}\right|+r\left(1_{\mathcal{Z}}\right)-\left|0_{\mathcal{Z}}\right|+|X|-r\left(1_{\mathcal{Z}}\right)-\left|E \backslash 1_{\mathcal{Z}}\right|+r(\bar{X})$ 
which can be simplified to

$$
r^{* *}(\bar{X})=|E|-\left|1_{\mathcal{Z}}\right|-\left|E \backslash 1_{\mathcal{Z}}\right|+r(\bar{X})
$$

which then obviously shows that

$$
r^{* *}(\bar{X})=r(\bar{X})
$$

Therefore the operation $r \mapsto r^{*}$ is self-inverse.

Lemma 83. The operation $r \mapsto r^{*}$ maps vertices of $P(\mathcal{Z})$ to vertices of $P\left(\mathcal{Z}^{*}\right)$.

Proof. Suppose that $r \in P(\mathcal{Z})$ is a vertex of $P(\mathcal{Z})$. Then there exist $n$ rows of the system $C(\mathcal{Z}) x \leq b(\mathcal{Z})$ where the inequalities are exact at $r$.

If $X_{1}, X_{2}$ be sets in $\mathcal{Z}$ such that $X_{2}$ covers $X_{1}$, and $r\left(X_{2}\right)-r\left(X_{1}\right)=1$, then $\bar{X}_{1}, \bar{X}_{2}$ are sets in $\mathcal{Z}^{*}$ where $\bar{X}_{1}$ covers $\bar{X}_{2}$. Then

$$
r\left(X_{1}\right)+1=r\left(X_{2}\right)
$$

can be transformed, by the subtraction of $r(M)$ from both sides, and by the addition of $\left|\bar{X}_{1}\right|-\left|\bar{X}_{1}\right|$ to the left-hand side and $\left|\bar{X}_{2}\right|-\left|\bar{X}_{2}\right|$ to the right side, into

$$
\left|\bar{X}_{1}\right|-r(M)+r\left(X_{1}\right)-\left|\bar{X}_{1}\right|+1=\left|\bar{X}_{2}\right|-r(M)+r\left(X_{2}\right)-\left|\bar{X}_{2}\right|
$$

which is equal to

$$
r^{*}\left(\bar{X}_{1}\right)-\left|\bar{X}_{1}\right|+1=r^{*}\left(\bar{X}_{2}\right)-\left|\bar{X}_{2}\right|
$$

which can then be rearranged to give

$$
r^{*}\left(\bar{X}_{1}\right)-r^{*}\left(\bar{X}_{2}\right)=\left|\bar{X}_{1}\right|-\left|\bar{X}_{2}\right|-1
$$

This then corresponds to an equality in the system $C\left(\mathcal{Z}^{*}\right) x \leq b\left(\mathcal{Z}^{*}\right)$.

If $Y_{1}, Y_{2}$ are sets in $\mathcal{Z}$ such that $Y_{2}$ covers $Y_{1}$, and

$$
r\left(Y_{2}\right)-r\left(Y_{1}\right)=\left|Y_{2}\right|-\left|Y_{1}\right|-1
$$


then $\bar{Y}_{1}, \bar{Y}_{2}$ are sets in $\mathcal{Z}^{*}$ where $\bar{Y}_{1}$ covers $\bar{Y}_{2}$. So

$$
r\left(Y_{2}\right)-r\left(Y_{1}\right)=\left|Y_{2}\right|-\left|Y_{1}\right|-1
$$

can be rearranged to give

$$
r\left(Y_{2}\right)-\left|Y_{2}\right|+1=r\left(Y_{1}\right)-\left|Y_{1}\right|,
$$

and the addition of $|E|-r(M)$ then gives

$$
|E|-\left|Y_{2}\right|-r(M)+r\left(Y_{2}\right)+1=|E|-\left|Y_{1}\right|-r(M)+r\left(Y_{1}\right)
$$

therefore

$$
\left|\bar{Y}_{2}\right|-r(M)+r\left(Y_{2}\right)+1=\left|\bar{Y}_{1}\right|-r(M)+r\left(Y_{1}\right)
$$

where the expressions for $r^{*}\left(\bar{Y}_{2}\right)$ and $r^{*}\left(\bar{Y}_{1}\right)$ can clearly be seen, meaning that

$$
r^{*}\left(\bar{Y}_{2}\right)+1=r^{*}\left(\bar{Y}_{1}\right) .
$$

This then corresponds to an equality in the system $C\left(\mathcal{Z}^{*}\right) x \leq b\left(\mathcal{Z}^{*}\right)$.

If $Z_{1}, Z_{2}$ are incomparable sets in $\mathcal{Z}$ such that

$$
r\left(Z_{1}\right)+r\left(Z_{2}\right)=r\left(Z_{1} \vee Z_{2}\right)+r\left(Z_{1} \wedge Z_{2}\right)+\left|Z_{1} \cap Z_{2}\right|-\left|Z_{1} \wedge Z_{2}\right|
$$

then $\bar{Z}_{1}, \bar{Z}_{2}$ are incomparable sets in $\mathcal{Z}^{*}$, and

$$
r\left(Z_{1}\right)+r\left(Z_{2}\right)=r\left(Z_{1} \vee Z_{2}\right)+r\left(Z_{1} \wedge Z_{2}\right)+\left|Z_{1} \cap Z_{2}\right|-\left|Z_{1} \wedge Z_{2}\right|
$$

implies that

$$
r\left(Z_{1}\right)+r\left(Z_{2}\right)=r\left(Z_{1} \vee Z_{2}\right)+r\left(Z_{1} \wedge Z_{2}\right)+\left|\bar{Z}_{1} \vee_{*} \bar{Z}_{2}\right|-\left|\bar{Z}_{1} \cup \bar{Z}_{2}\right|
$$

and adding $\left|\bar{Z}_{1}\right|+\left|\bar{Z}_{1}\right|$ to both sides then gives

$r\left(Z_{1}\right)+r\left(Z_{2}\right)+\left|\bar{Z}_{1}\right|+\left|\bar{Z}_{2}\right|=r\left(Z_{1} \vee Z_{2}\right)+r\left(Z_{1} \wedge Z_{2}\right)+\left|\bar{Z}_{1} \vee_{*} \bar{Z}_{2}\right|+\left|\bar{Z}_{1} \cap \bar{Z}_{2}\right|$.

Then

$r\left(Z_{1}\right)+r\left(Z_{2}\right)+\left|\bar{Z}_{1}\right|+\left|\bar{Z}_{2}\right|=r\left(\bar{Z}_{1} \wedge_{*} \bar{Z}_{2}\right)+r\left(\bar{Z}_{1} \vee_{*} \bar{Z}_{2}\right)+\left|\bar{Z}_{1} \vee_{*} \bar{Z}_{2}\right|+\left|\bar{Z}_{1} \cap \bar{Z}_{2}\right|$ 
which in turn implies that

$$
\begin{aligned}
& \left|\bar{Z}_{1}\right|+r\left(Z_{1}\right)+\left|\bar{Z}_{2}\right|+r\left(Z_{2}\right)= \\
& \left|\bar{Z}_{1} \vee_{*} \bar{Z}_{2}\right|+r\left(\bar{Z}_{1} \vee_{*} \bar{Z}_{2}\right)+\left|\bar{Z}_{1} \wedge_{*} \bar{Z}_{2}\right|+r\left(\bar{Z}_{1} \wedge_{*} \bar{Z}_{2}\right)+\left|\bar{Z}_{1} \cap \bar{Z}_{2}\right|-\left|\bar{Z}_{1} \wedge_{*} \bar{Z}_{2}\right|
\end{aligned}
$$

therefore

$r^{*}\left(\bar{Z}_{1}\right)+r^{*}\left(\bar{Z}_{2}\right)=r^{*}\left(\bar{Z}_{1} \vee_{*} \bar{Z}_{2}\right)+r^{*}\left(\bar{Z}_{1} \wedge_{*} \bar{Z}_{2}\right)+\left|\bar{Z}_{1} \cap \bar{Z}_{2}\right|-\left|\bar{Z}_{1} \wedge_{*} \bar{Z}_{2}\right|$

This then corresponds to an equality in the system $C\left(\mathcal{Z}^{*}\right) x \leq b\left(\mathcal{Z}^{*}\right)$.

The rows restricting $0_{\mathcal{Z}}$ to a rank of no more than (and no less than) zero will be equalities in $C(\mathcal{Z}) x \leq b(\mathcal{Z})$. Similarly, the rows restricting $0_{\mathcal{Z}^{*}}$ to a rank of no more than (and no less than) zero will be equalities in $C\left(\mathcal{Z}^{*}\right) x \leq b\left(\mathcal{Z}^{*}\right)$.

Therefore, for every distinct equality in the system $C(\mathcal{Z}) x \leq b(\mathcal{Z})$ at the point $r$, there is a corresponding, unique equality in the system $C\left(\mathcal{Z}^{*}\right) x \leq$ $b\left(\mathcal{Z}^{*}\right)$ at the point $r^{*}$. A set of rows in $C\left(\mathcal{Z}^{*}\right)$ is linearly independent if and only if the corresponding rows in $C(\mathcal{Z})$ are linearly independent, so, as $r^{*} \in P\left(\mathcal{Z}^{*}\right)$, then $r^{*}$ is a vertex of $P\left(\mathcal{Z}^{*}\right)$.

Corollary 84. Classes III, IV, and V are closed under duality.

Proof. This follows directly from Theorem 81 and from Lemmas 82 and 83 . The rank function is a bijection between the two polyhedra, where integral vertices are mapped to integral vertices, and nonintegral vertices to nonintegral vertices, and, trivially, integral points are mapped to integral points. Therefore if any lattice $\mathcal{Z}$ belongs to any of classes III, IV, or $\mathrm{V}$, its dual must also belong to that class. 


\section{Chapter 6}

\section{Lattice minors}

\subsection{Defining lattice minors}

In this chapter, we construct a definition of lattice minors inspired by matroid minors, and characterise class-I lattices in terms of their minors.

Unlike the free product or direct sum operations on matroids, when given a lattice of sets $\mathcal{Z}$, there is no well-defined transformation that will occur when taking a minor of a matroid that has $\mathcal{Z}$ as its lattice of cyclic flats. This is because the lattice of cyclic flats can change in different ways depending on the ranks assigned.

For example, consider the matroids in Figure 6.1 .1 on page 58 and Figure 6.1.2 on page 58. Each has the same lattice of cyclic flats (Figure 6.1.3). But the matroids obtained by deleting the element $e$ from each have different lattices of cyclic flats (compare Figures 6.1.4 and 6.1.5).

However, we know from Lemma 51 that, for any matroid $M$ and element $e$ of $E(M)$, that if $Z$ is a cyclic flat of $M \backslash e$, then exactly one of $Z$ and $Z \cup\{e\}$ is a cyclic flat of $M$. Then clearly there is an order-preserving injection from $\mathcal{Z}(M \backslash e)$ to $\mathcal{Z}(M)$. As contraction is the dual operation of deletion in minors, and the dual of a lattice of sets possesses the same lattice structure with order relations reversed, contraction of matroid elements must then affect the lattice of cyclic flats in a fundamentally similar way. This is 


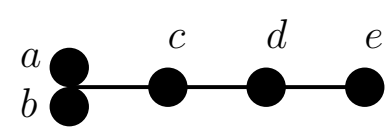

Figure 6.1.1: $M_{1}$
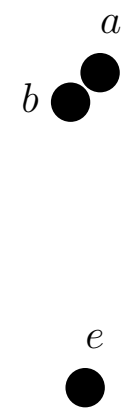

Figure 6.1.2: $M_{2}$
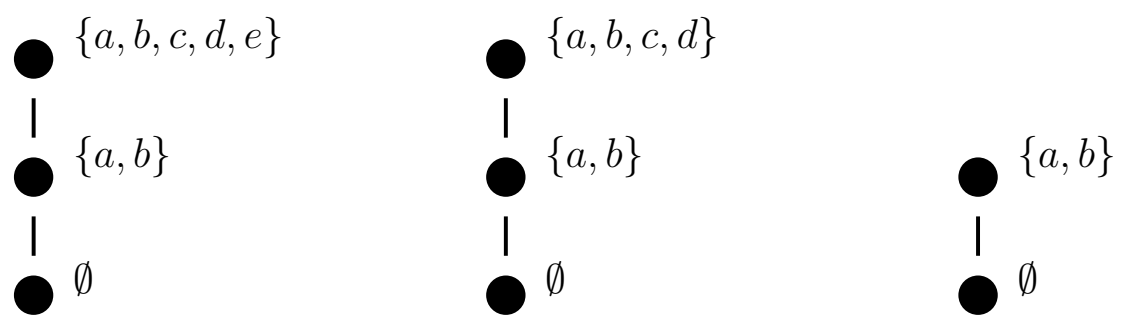

Figure 6.1.3: Cyclic Figure 6.1.4: Cyclic Figure 6.1.5: Cyclic flats of both $M_{1}$ and flats of $M_{1} \backslash e$ flats of $M_{2} \backslash e$ $M_{2}$ 


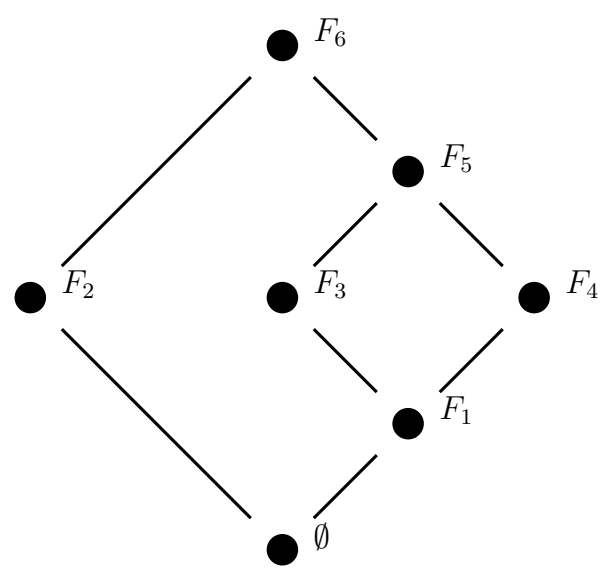

Figure 6.1.6: A lattice of sets

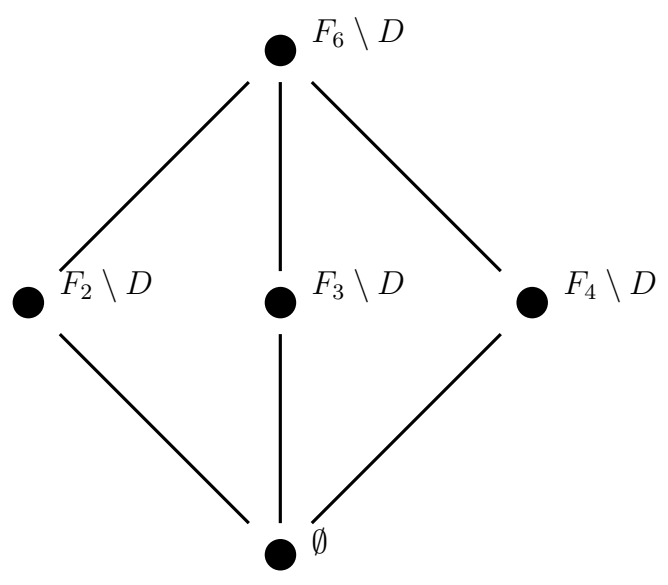

Figure 6.1.7: A lattice minor of the lattice in Figure 6.1.6

the inspiration for the following definitions.

Definition 85. If $\mathcal{Z}$ is a lattice of sets on $E$, then $\mathcal{X}$ is a lattice minor of $\mathcal{Z}$ if $\mathcal{X}$ is a nonempty lattice of sets, and there exist some $\mathcal{W} \subseteq \mathcal{Z}$ and $D \subseteq E$ such that $\mathcal{X}=\{W \backslash D: W \in \mathcal{W}\}$ and there is a lattice isomorphism from $\mathcal{W}$ to $\mathcal{X}$.

Definition 86. If $\mathcal{Z}$ is a lattice of sets on $E$, and $\mathcal{X}$ is a lattice minor of $\mathcal{Z}$, then there exists some $\mathcal{W} \subseteq \mathcal{Z}$ that is lattice-isomorphic to $\mathcal{X}$, and some $D \subseteq E$ such that $\mathcal{X}=\{W \backslash D: W \in \mathcal{W}\}$. Then given any $W \in \mathcal{W}$, $X=W \backslash D$ for some $X \in \mathcal{X}$, and we call $W$ the preimage of $X$ in $\mathcal{Z}$, denoted ${ }_{\mathcal{Z}} X$, and $X$ the image of $W$ in $\mathcal{X}$, denoted $W_{\mathcal{X}}$. Given any $S \subseteq \mathcal{Z}$, call $S_{\mathcal{X}}=\left\{X \in \mathcal{X}:{ }_{\mathcal{Z}} X \in S\right\}$ the image of $S$ in $\mathcal{X}$.

Example 87. Figure 6.1.7 on page 59 is a lattice minor of Figure 6.1.6.

Though it is not necessarily the case that every lattice minor of a matroidal lattice will be matroidal, the lattice of cyclic flats of a matroid $M \backslash e$ or $M / e$ will be a lattice minor of the lattice of cyclic flats of $M$. 


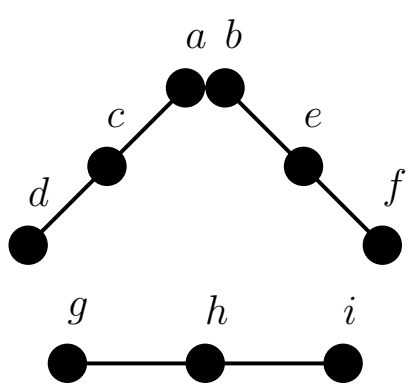

Figure 6.1.8: A matroid $N$

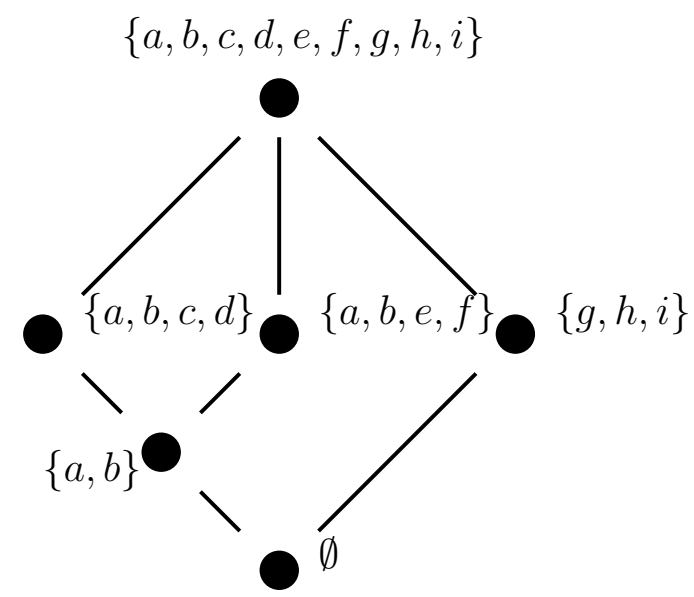

Figure 6.1.9: Lattice of cyclic flats of $N$

Proposition 88. Let $\mathcal{Z}$ be a lattice of sets on $E$, and let $S, T \subseteq \mathcal{Z}$ such that $\bigvee_{\mathcal{Z}} S \leq \bigwedge_{\mathcal{Z}} T$. Let $\mathcal{X}$ be a lattice minor of $\mathcal{Z}$. Then $\bigvee_{\mathcal{X}} S_{\mathcal{X}} \leq \bigwedge_{\mathcal{X}} T_{\mathcal{X}}$.

Proof. Suppose there exist some $\mathcal{Z}, \mathcal{X}, S$ and $T$ as described above, where $\bigvee_{\mathcal{X}} S_{\mathcal{X}}>\bigwedge_{\mathcal{X}} T_{\mathcal{X}}$. Order relations are preserved when taking lattice minors of lattices. Therefore, for any $s \in S_{\mathcal{X}}$ and $t \in T_{\mathcal{X}}, s \leq t$. So every $t \in T_{\mathcal{X}}$ is an upper bound for $S_{\mathcal{X}}$. Then, as $\bigvee_{\mathcal{X}} S_{\mathcal{X}}>\wedge_{\mathcal{X}} T_{\mathcal{X}}$, there exists some $t \in T_{\mathcal{X}}$ such that $\bigvee_{\mathcal{X}} S_{\mathcal{X}} \not \leq t$. Therefore $t$ and $\bigvee_{\mathcal{X}} S_{\mathcal{X}}$ are incomparable upper bounds for $S_{\mathcal{X}}$. This contradicts the fact that $\bigvee_{\mathcal{X}} S_{\mathcal{X}}$ is the least upper bound for $S_{\mathcal{X}}$

Definition 89. An embedding of a lattice $\mathcal{X}$ into a lattice $\mathcal{Z}$ is an injection $\phi: \mathcal{X} \rightarrow \mathcal{Z}$ such that

$$
\begin{aligned}
& \phi(X) \vee_{\mathcal{Z}} \phi(Y)=\phi\left(X \vee_{\mathcal{X}} Y\right) \\
& \phi(X) \wedge_{\mathcal{Z}} \phi(Y)=\phi\left(X \wedge_{\mathcal{X}} Y\right)
\end{aligned}
$$

for all $X, Y \in \mathcal{X}$. 


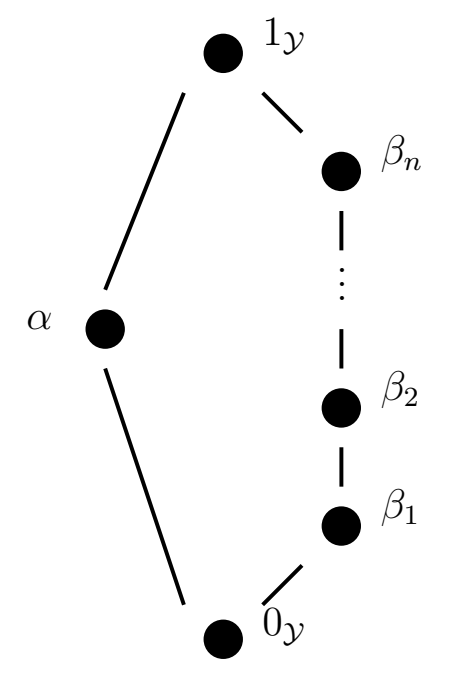

Figure 6.2.1: The lattice $\mathcal{Y}_{n}$

Proposition 90. The preimage $\mathcal{Z}^{\mathcal{X}}$ of a lattice minor $\mathcal{X}$ of a lattice $\mathcal{Z}$ need not be embeddable in $\mathcal{Z}$.

Proof. Consider the matroid $N$ in Figure 6.1 .8 on page 60. Its lattice of cyclic flats is shown in Figure 6.1.9. Then consider the matroid $N \backslash a$ and $\mathcal{X}=\mathcal{Z}(N \backslash a)$. Examining how the cyclic flats change under this deletion, it is clear that the cyclic flat $\{a, b\}$ is removed from the lattice of cyclic flats, while $\{a, b, c, d\}$ and $\{a, b, e, f\}$ are transformed to $\{b, c, d\}$ and $\{b, e, f\}$, respectively. So the preimage of $\mathcal{Z}(N \backslash a)$ is ${ }_{\mathcal{Z}(N)} \mathcal{X}=\mathcal{Z}(N) \backslash\{\{a, b\}\}$. This lattice is not embeddable in $\mathcal{Z}(N)$, as it contains $\{a, b, c, d\}$ and $\{a, b, e, f\}$, yet their meet in $\mathcal{Z}(N)$ is $\{a, b\}$, which is not contained in ${ }_{\mathcal{Z}(N)} \mathcal{X}$.

\subsection{Minor-closed classes of lattice}

Lemma 91. A lattice of the form depicted in Figure 6.2.1 on page 61 belongs to class I. 
Proof. By Proposition 44, a matrix is totally unimodular if and only if, given any collection of columns $\left\{Y_{1}, Y_{2}, \ldots, Y_{t}\right\}$ of the matrix, there exists a sequence $\left(\epsilon_{j}\right)_{j \leq t}$, where $\epsilon_{j}= \pm 1$ for all $1 \leq j \leq t$, such that $\sum_{i=1}^{t} \epsilon_{i} Y_{i}$ is a totally unimodular vector.

Let $L$ be a lattice of the form depicted in Figure 6.2.1, and $\mathcal{C}$ the constraint matrix constructed from it. Given a collection $Y=\left\{Y_{1}, Y_{2}, \ldots, Y_{t}\right\}$ of columns of $\mathcal{C}$, construct a sequence $\left(\epsilon_{j}\right)_{j \leq t}$, where $\epsilon_{j}= \pm 1$ for all $1 \leq j \leq t$, that adheres to the following rules:

1. If $Y_{p}$ covers $Y_{q}$, then $\epsilon_{p}=\epsilon_{q}$.

2. If $Y_{s}=0_{\mathcal{Y}} \in Y$ and $Y_{r}=1_{\mathcal{Y}} \in Y$, but there is no chain of cover relations in $Y$ connecting $Y_{s}$ and $Y_{r}$, then $\epsilon_{s}=-\epsilon_{r}$.

3. If $0 y, 1 \mathcal{Y}, \alpha \in Y$, then $\epsilon_{j}=\epsilon l$ for all $1 \leq j, l \leq t$.

4. If only one of $0_{\mathcal{Y}}$ and $1_{\mathcal{Y}}$ is in $Y$, then $\epsilon_{j}=\epsilon l$ for all $1 \leq j, l \leq t$.

5. If $Y_{m}=\alpha \in Y$, but $0_{\mathcal{Y}}, 1_{\mathcal{Y}} \notin Y$, then $\epsilon_{j} \neq \epsilon_{m}$ for all $j \neq m$.

We will show that, given such a sequence, $\sum_{i=1}^{t} \epsilon_{i} Y_{i}$ gives a totally unimodular vector.

It is easy to see that these rules are noncontradictory, so it remains to show that in every row the sum will be $-1,0$, or 1 .

Clearly, the rows of $C\left(\mathcal{Y}_{n}\right)$ defining the rank of the 0 element will return a sum of 0,1 , or -1 regardless of the choice of sequence, as there is only one nonzero entry in each of these rows.

Then consider a cover relation row. There are two nonzero entries. Say these are at locations $p$ and $q$. If at most one of these columns is in $Y$, the particular choice of sequence is irrelevant, and the sum is totally unimodular. If both of these columns are in $Y$, then by rule 1 above, $\epsilon_{p}=\epsilon_{q}$, and so the entries of this row sum to 0 , meaning the choice of sequence is valid for this row.

Consider a weight- 4 row. Any incomparable pair must consist of $\alpha$ and $\beta_{k}$ for some $1 \leq k \leq n$, with meet and join $0_{\mathcal{Y}}$ and $1_{\mathcal{Y}}$, respectively. 
If at most one of the nonzero columns is in $Y$, the choice of sequence is trivially valid for this row.

Then suppose there are two nonzero columns in $Y$. If those two columns are $Y_{s}=0_{\mathcal{Y}}$ and $Y_{r}=1_{\mathcal{Y}}$, then $\alpha \notin Y$ and $\beta_{k} \notin Y$ for some $1 \leq k \leq n$, therefore there is no chain of cover relations connecting $0_{\mathcal{Y}}$ and $1_{\mathcal{Y}}$; therefore, $\epsilon_{s}=-\epsilon_{r}$, meaning the value at one location is subtracted from the other in the sum, and, as both entries are 1 in this row, the choice of sequence is valid for this row. If those two columns are $\beta_{k}$ (where $1 \leq k \leq n$ ) and $0_{\mathcal{Y}}$, then, as only one of $0_{\mathcal{Y}}$ and $1_{\mathcal{Y}}$ is in $Y, \epsilon_{j}=\epsilon_{l}$ for all $j, l$, meaning the $\beta_{k}$ and $0 y$ entries are added to each other; as the entries in the $\beta_{k}$ and $0_{y}$ columns have opposite sign in this row, the sequence is valid for this row. Similar reasoning applies if the two nonzero columns in $Y$ are $\beta_{k}$ and $1_{\mathcal{Y}}$, or $\alpha$ and $0 \mathcal{y}$, or $\alpha$ and $1_{\mathcal{Y}}$. If the two columns are $Y_{m}=\alpha$ and $\beta_{k}$, then, as $0_{\mathcal{Y}}, 1_{\mathcal{Y}} \notin Y$, $\epsilon_{j} \neq \epsilon_{m}$ for all $j \neq m$, meaning that one entry is subtracted from the other in the sum; as $\alpha$ and $\beta_{k}$ have the same sign in this row, this will equal zero, making the sequence valid for this row.

Then suppose there are three nonzero columns in $Y$. If those three are $0 \mathcal{y}, \alpha, \beta_{k}$ or $\alpha, \beta_{k}, 1 \mathcal{Y}$, then all values of $\epsilon_{j}$ are the same, meaning they are all added to one another; this sum will equal \pm 1 , meaning the sequence is valid for this row. If those three are $0_{\mathcal{Y}}, \alpha, 1_{\mathcal{Y}}$, then all values of $\epsilon_{j}$ are the same; this sum will equal \pm 1 . If those three are $0_{\mathcal{Y}}, \beta_{k}, 1_{\mathcal{Y}}$, then either there is a chain of cover relations in $Y$ connecting $0_{\mathcal{y}}$ and $1_{\mathcal{y}}$ or there is not; if there is, then all values values of $\epsilon_{j}$ are the same, and that row sums to \pm 1 , while if there is not, then letting $\beta_{k}=Y_{u}, \epsilon_{u}$ is equal to exactly one of $\epsilon_{s}$ and $\epsilon_{r}$, meaning that the sum across that row is \pm 1 , and so the choice of sequence is valid for the row.

If all four nonzero columns are in $Y$, then values of $\epsilon_{j}$ are the same, and this row is summed to 0 , meaning that the choice of sequence is valid for this row.

Theorem 92. A lattice $\mathcal{Z}$ belongs to class I if and only if $\mathcal{Z}$ has no lattice minor isomorphic to the lattices in Figures 6.2.2 to 6.2.4. 


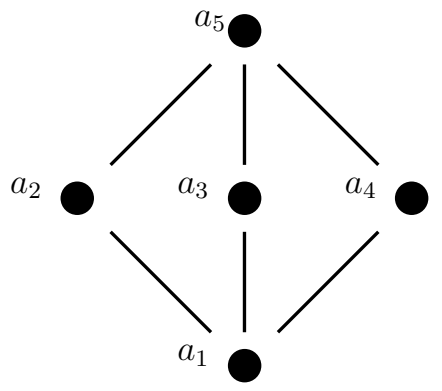

Figure 6.2.2:

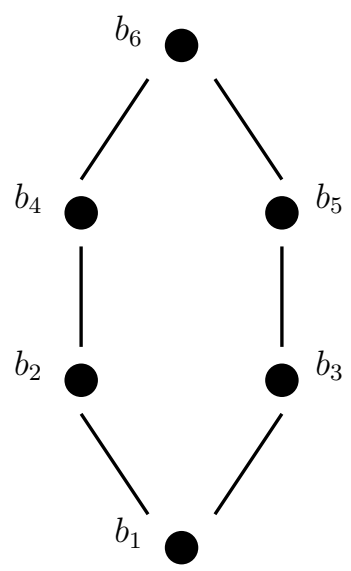

Figure 6.2.3:

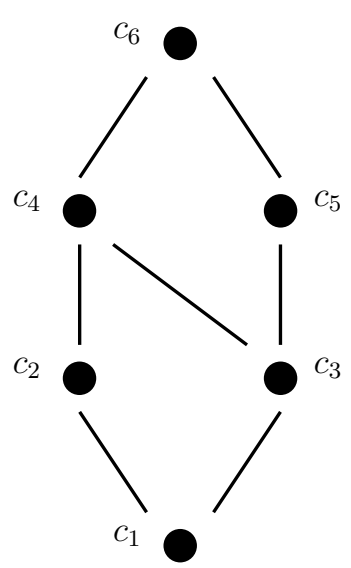

Figure 6.2.4:

Proof. Suppose $\mathcal{Z}$ has a lattice minor isomorphic to the lattice depicted in Figure 6.2.2. Then $\mathcal{Z}$ has an antichain with 3 elements. Let $Z_{1}, Z_{2}$, and $Z_{3}$ be mutually incomparable sets in $\mathcal{Z}$. Then $C(\mathcal{Z})$ has the following submatrix

$$
\begin{array}{ccc}
Z_{1} & Z_{2} & Z_{3} \\
1 & 1 & 0 \\
1 & 0 & 1 \\
0 & 1 & 1
\end{array}
$$

which has determinant -2 .

Suppose $\mathcal{Z}$ has a lattice minor isomorphic to the lattice depicted in Figure 6.2.3. Then there is some $\mathcal{W} \subseteq \mathcal{Z}$ that is isomorphic to Figure 6.2.3. Select some such $\mathcal{W}=\left\{Z_{1}, Z_{2}, Z_{3}, Z_{4}, Z_{5}, Z_{6}\right\}$, where there is a lattice isomorphism that maps $Z_{i}$ to $b_{i}$, such that $Z_{2}$ and $Z_{3}$ cover $Z_{1}$, and $Z_{1}$ and $Z_{6}$ are the meet and join, respectively, of $Z_{2}$ and $Z_{3}$, as well as of $Z_{4}$ and $Z_{5}$. Then $C(\mathcal{Z})$ has the following submatrix

$$
\begin{array}{cccc}
Z_{1} & Z_{2} & Z_{3} & Z_{6} \\
-1 & 1 & 0 & 0 \\
-1 & 1 & 1 & -1 \\
-1 & 0 & 0 & -1 \\
-1 & 0 & 1 & 0
\end{array}
$$


which has determinant -2 .

Suppose $\mathcal{Z}$ has a lattice minor of the form shown in Figure 6.2.4. Then there is some $\mathcal{W} \subseteq \mathcal{Z}$ that is isomorphic to Figure 6.2.4. Select some such $\mathcal{W}=\left\{Z_{1}, Z_{2}, Z_{3}, Z_{4}, Z_{5}, Z_{6}\right\}$, where there is a lattice isomorphism that maps $Z_{i}$ to $c_{i}$, such that $Z_{4}$ covers both $Z_{2}$ and $Z_{3}$, and $Z_{5}$ covers $Z_{3}$. Then $C(\mathcal{Z})$ has the following submatrix

$\begin{array}{cccc}Z_{2} & Z_{3} & Z_{4} & Z_{5} \\ 1 & 0 & 0 & 1 \\ -1 & 0 & 1 & 0 \\ 0 & -1 & 1 & 0 \\ 0 & -1 & 0 & 1\end{array}$

which has determinant 2 .

Suppose that $\mathcal{Z}$ has no lattice minor isomorphic to any of these cases. Then it must have width no more than 2 . If $\mathcal{Z}$ has width 1 , it is a chain, and $C(\mathcal{Z})$ is totally unimodular, therefore $\mathcal{Z}$ belongs to class $\mathrm{I}$.

So then suppose $\mathcal{Z}$ has width 2 . Let $S$ and $T$ be distinct maximal chains in $\mathcal{Z}$, and let $s_{1}$ and $s_{2}$ be distinct elements of $S \cap T$ such that $s_{1}<s_{2}$ and there is no $s_{3} \in S \cap T$ where $s_{1}<s_{3}<s_{2}$. Then there must exist some $x \in S$ or $x \in T$ such that $x$ covers $s_{1}$ and $s_{2}$ covers $x$, for, if there is not, then $\mathcal{Z}$ has a subcollection $\mathcal{W}$ isomorphic to either Figure 6.2.3 or Figure 6.2.4, which contradicts the assumption that neither of those is a lattice minor of $\mathcal{Z}$.

Then $\mathcal{Z}$ must be a free product (linear sum) of chains and of lattices of the form of Figure 6.2.1 on page 61. As shown in Theorem 72, the free product operation preserves class I membership, therefore, since both such families of lattice belong to class I (by Lemma 91), so too does $\mathcal{Z}$.

Corollary 93. Class I is closed under taking lattice minors.

Proof. A class I lattice $\mathcal{Z}$ has no lattice minor isomorphic to the lattices depicted in Figures 6.2.2 to 6.2.4. So there is no $\mathcal{W} \subseteq \mathcal{Z}$ isomorphic to any of these. Therefore no lattice minor of $\mathcal{Z}$ has a lattice minor isomorphic to 
Figures 6.2.2 to 6.2.4. Therefore every lattice minor of $\mathcal{Z}$ belongs to class I.

Lemma 94. Class III is not closed under taking lattice minors.

Proof. Consider the lattice of sets $\mathcal{Z}_{1}$ shown in Figure 6.2 .5 on page 67 (found by a random computer search). The sets in this lattice are

$$
\begin{aligned}
& d_{1}=\{1,3,5,6,11,12\} \\
& d_{2}=\{0,1,5,6,9,10,13\} \\
& d_{3}=\{0,6,7,8,10,12,13\} \\
& d_{4}=\{1,4,6,7,8,10,13\} \\
& d_{5}=\{0,2,3,5,7,8,9,11,13\} \\
& d_{6}=\{1,2,3,5,6,11,12,13\} \\
& E=\{0,1,2,3,4,5,6,7,8,9,10,11,12,13\} .
\end{aligned}
$$

This lattice is matroidal, and $P\left(\mathcal{Z}_{1}\right)$ has two vertices:

$$
\begin{aligned}
& r_{1}=(0,5,6,6,6,6,6,7) \\
& r_{2}=(0,5,5,6,6,6,6,7)
\end{aligned}
$$

therefore $\mathcal{Z}_{1}$ belongs to class III.

Consider the matroid $M_{1}$ defined by the rank assignment $r_{2}$. If the element 2 is deleted from this matroid, the resulting lattice of cyclic flats is shown in Figure 6.2.6 on page 68. It is easy to see that $\mathcal{Z}_{1}^{\prime}$ is a lattice minor of $\mathcal{Z}_{1}$. The vertices of $P\left(\mathcal{Z}_{1}^{\prime}\right)$ are

$$
\begin{aligned}
r_{1}^{\prime} & =(0,5,5,6,6,6,7) \\
r_{2}^{1} & =(0,5,6,6,6,6,7) \\
r_{3}^{\prime} & =(0,4,6,6,6,6,7) \\
r_{4}^{\prime} & =(0,5,6,6,6,5,7) \\
r_{5}^{\prime} & =\left(0, \frac{9}{2}, \frac{11}{2}, 6,6, \frac{11}{2}, 7\right)
\end{aligned}
$$




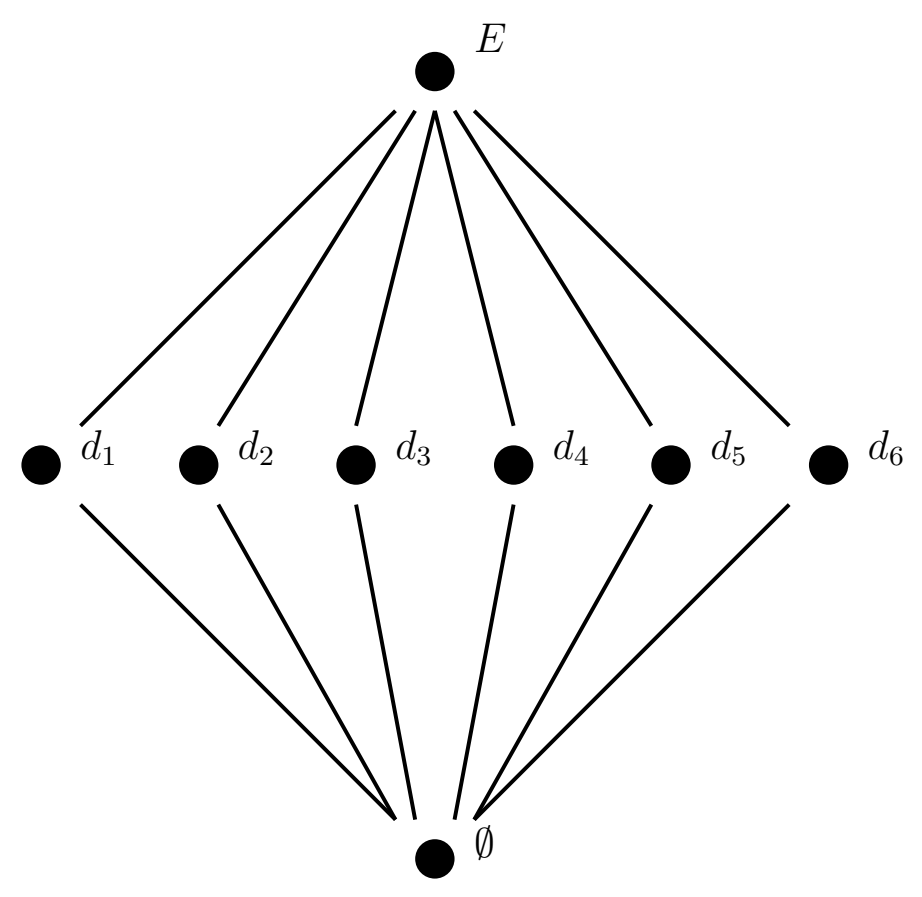

Figure 6.2.5: A lattice $\mathcal{Z}_{1}$ 


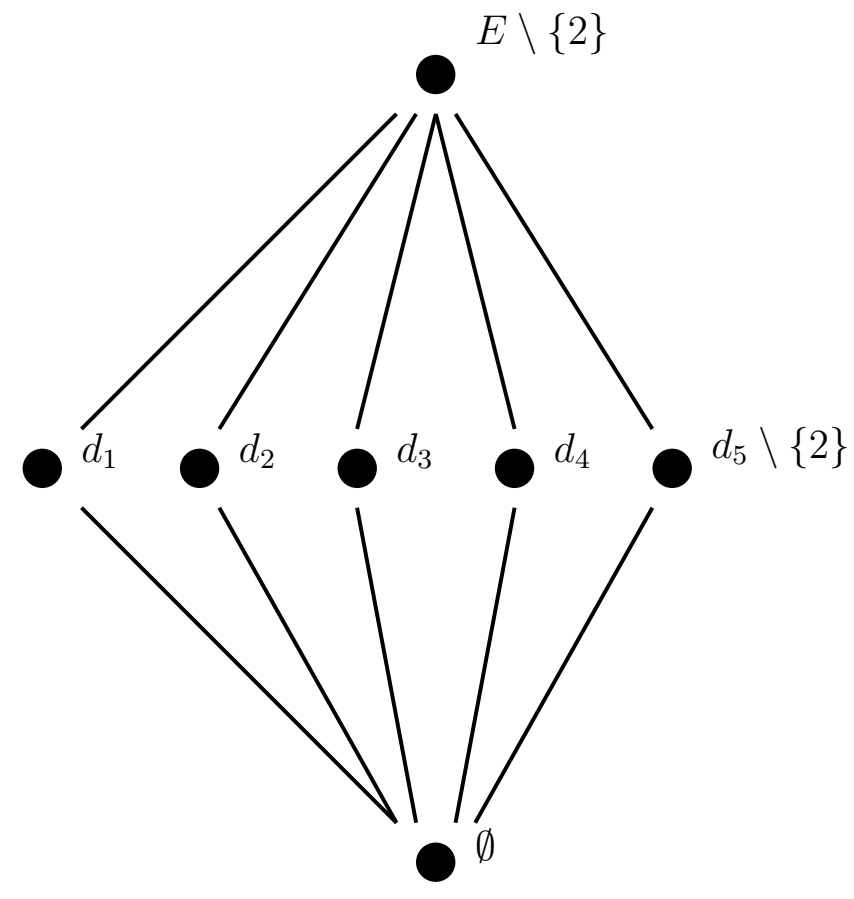

Figure 6.2.6: A lattice minor $\mathcal{Z}_{1}^{\prime}$ of $\mathcal{Z}_{1}$ 
therefore $\mathcal{Z}_{1}^{\prime}$ does not belong to class III. Therefore class III is not closed under taking minors. 
Appendices 



\section{Appendix A}

\section{Software}

The Sage software package was used to analyse matroids and lattices of sets.

The following code implements the basic lattice operations meet and join, and can determine if one set covers another in a given lattice of sets. This is necessary for subsequent code.

1 def Join(Fone,Ftwo,L):

$2 \quad \mathrm{E}=\operatorname{set}()$

3 for $\mathrm{F}$ in $\mathrm{L}$ :

$4 \quad \mathrm{E}=\mathrm{E} \cdot \operatorname{union}(\mathrm{F})$

${ }_{5} \quad \mathrm{U}=$ Fone.union(Ftwo)

$6 \quad$ Best $=\mathrm{E}$

7 for $\mathrm{F}$ in $\mathrm{L}$ :

$8 \quad$ if U.issubset(F) and F.issubset(Best):

$9 \quad$ Best $=\mathrm{F}$

10 return Best

11

12 def Meet(Fone,Ftwo,L):

$13 \quad \mathrm{I}=$ Fone.intersection(Ftwo)

${ }_{14} \quad$ Best $=\operatorname{set}()$

15 for $\mathrm{F}$ in $\mathrm{L}$ :

16 if F.issubset(I) and Best.issubset(F): 


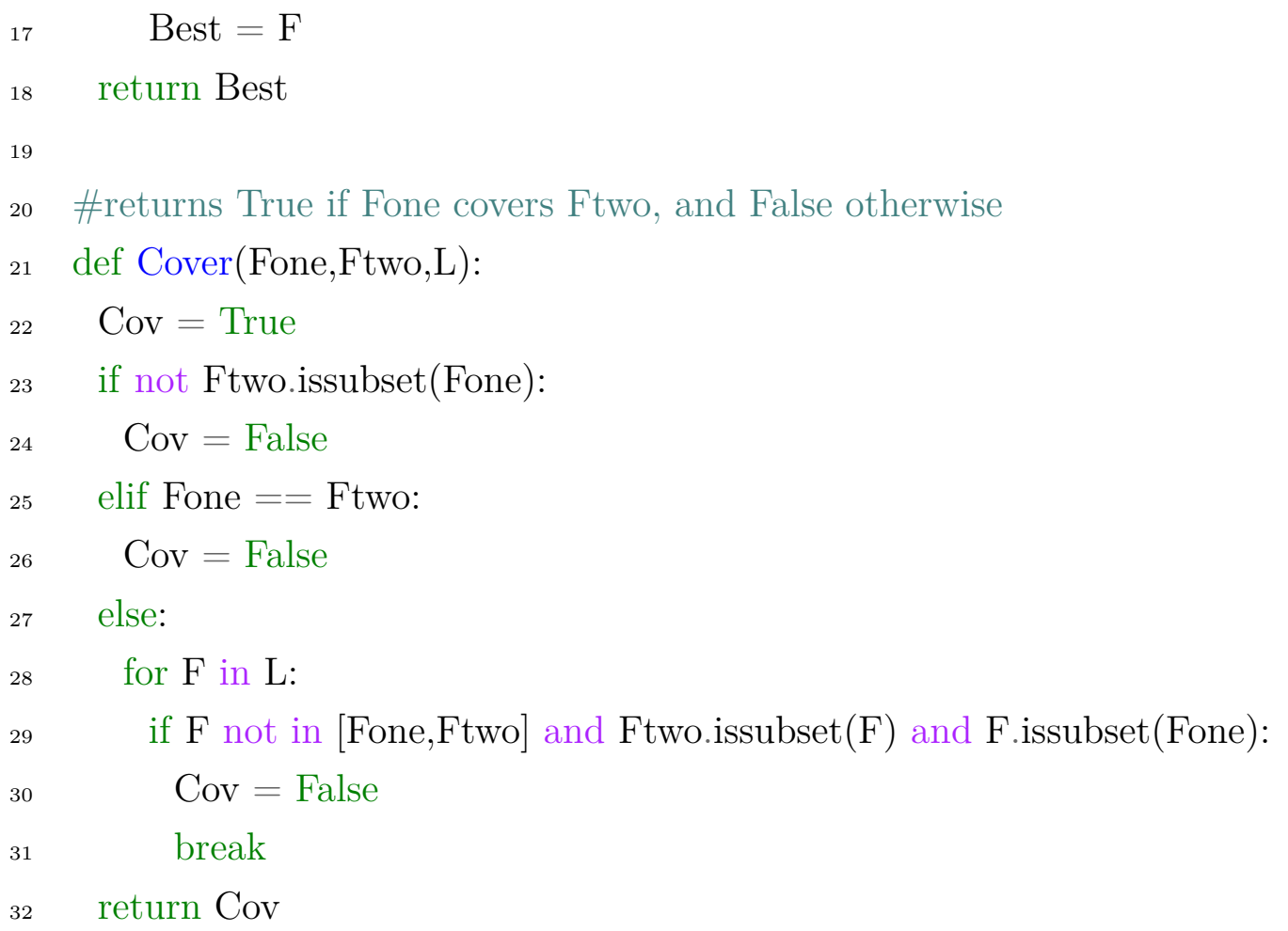

The Poly function was used to generate a polyhedron from a list of cyclic flats. Typically, this was done in order to examine the vertices of the polyhedron.

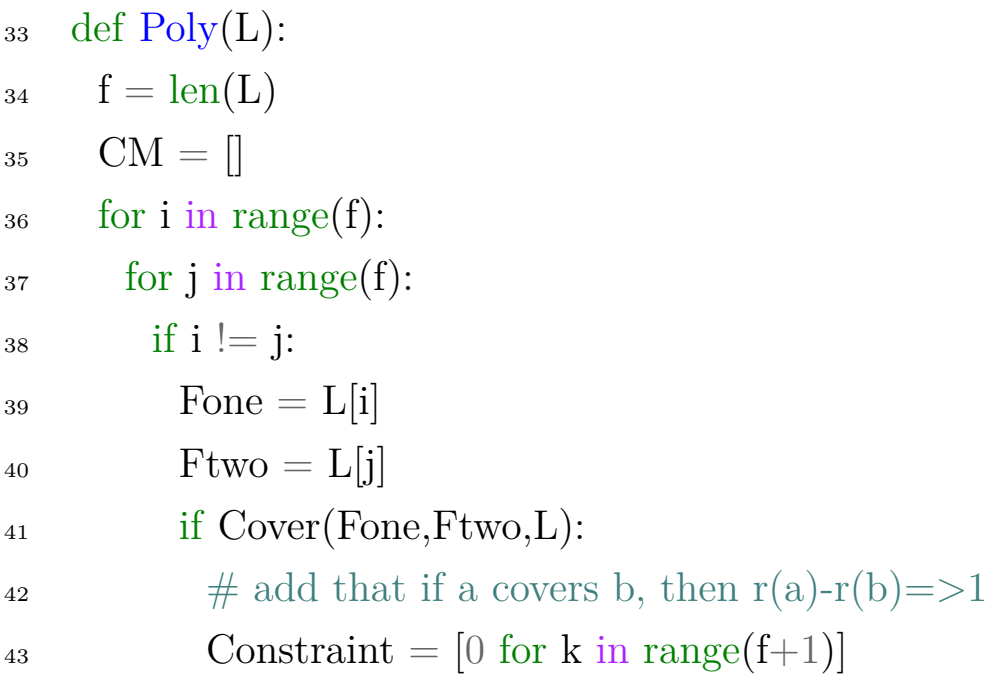




\section{APPENDIX A. SOFTWARE}

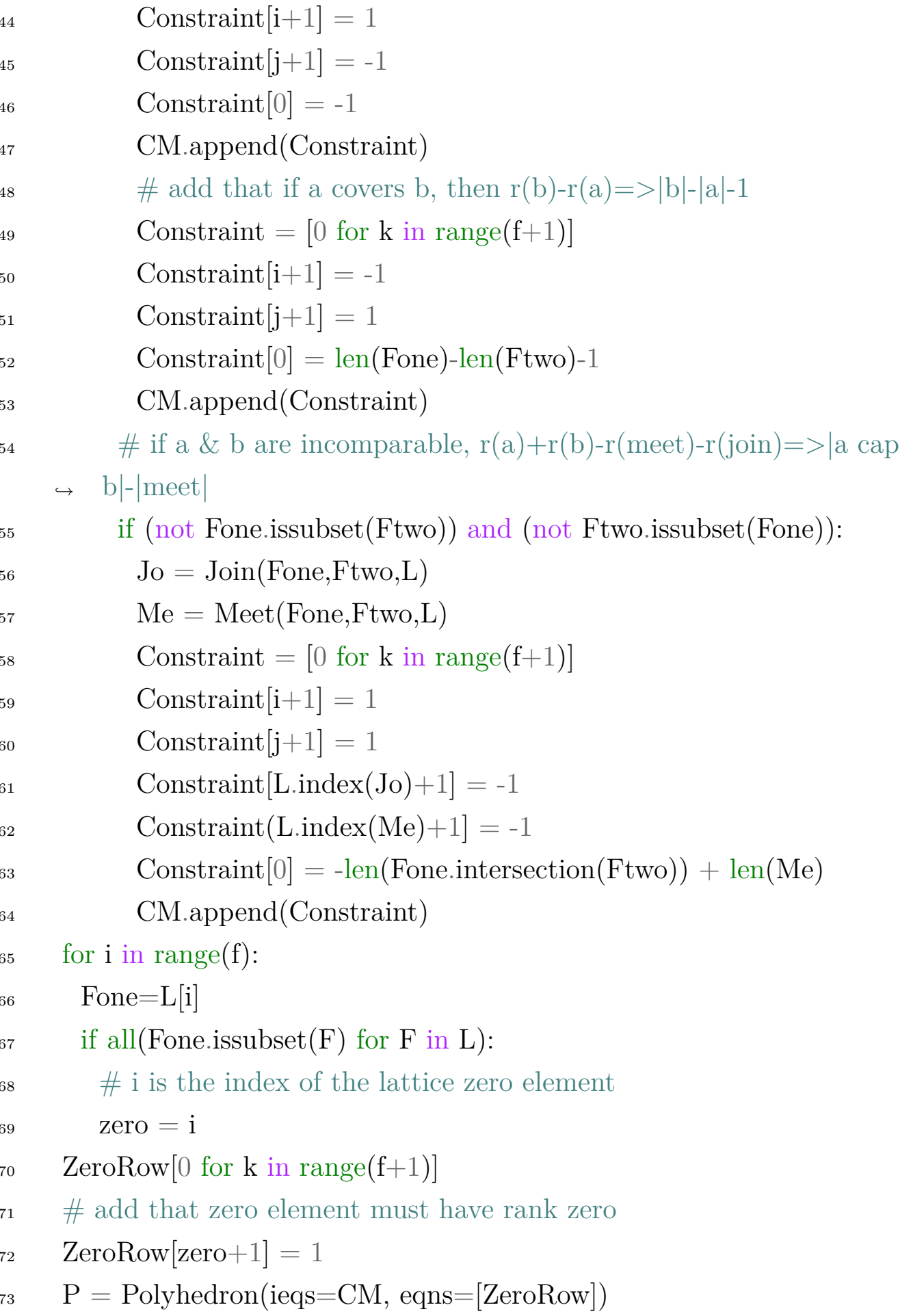


74 return $\mathrm{P}$

A typical use case of the Poly function was

$75 \mathrm{~L}=[\operatorname{set}(),\{1,2,3\},\{4,5,6\},\{6,7,1\},\{1,2,3,4,5,6,7\}]$

76 $\mathrm{P}=\operatorname{Poly}(\mathrm{L})$

77 list(P.vertex_generator ()$)$

78

$79 \quad \# \#[$ A vertex at $(0,2,2,2,3)]$

The Poly function was also adapted into the LMat function, used to generate a matrix representing the system of inequalities, or, more commonly, a reduced form of the constraint matrix alone, in which, if a pair of rows were linearly dependent, one of them was removed.

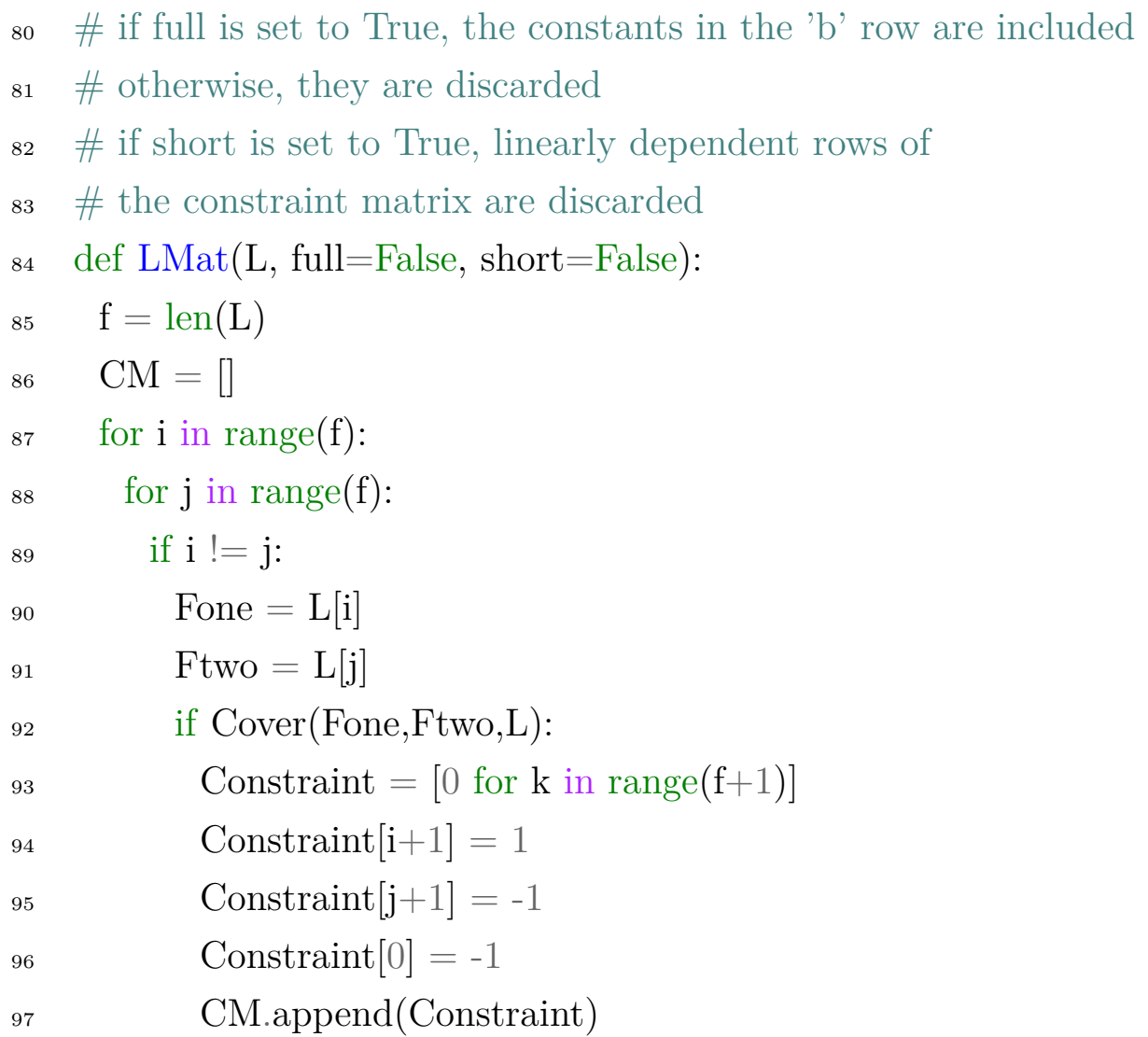


if not short:

Constraint $=[0$ for $\mathrm{k}$ in range $(\mathrm{f}+1)]$

Constraint $[\mathrm{i}+1]=-1$

Constraint $[\mathrm{j}+1]=1$

Constraint[0] = len(Fone)-len(Ftwo)-1

CM.append(Constraint)

if (not Fone.issubset(Ftwo)) and (not Ftwo.issubset(Fone)):

$\mathrm{Jo}=\operatorname{Join}($ Fone,Ftwo,L)

$\mathrm{Me}=\operatorname{Meet}($ Fone,Ftwo,L)

Constraint $=[0$ for $\mathrm{k}$ in range $(\mathrm{f}+1)]$

Constraint $[\mathrm{i}+1]=1$

Constraint $[\mathrm{j}+1]=1$

Constraint $\left[\mathrm{L} \cdot \operatorname{index}\left(\mathrm{Jo}_{\mathrm{O}}\right)+1\right]=-1$

Constraint(L.index $(\mathrm{Me})+1]=-1$

Constraint $[0]=-\operatorname{len}($ Fone.intersection $($ Ftwo $))+\operatorname{len}(\mathrm{Me})$

CM.append(Constraint)

for $i$ in range(f):

Fone $=\mathrm{L}[\mathrm{i}]$

if all(Fone.issubset(F) for $\mathrm{F}$ in $\mathrm{L}$ ):

zero $=\mathrm{i}$

ZeroRow $[0$ for $\mathrm{k}$ in range $(\mathrm{f}+1)]$

ZeroRow $[$ zero +1$]=1$

CM.append(ZeroRow)

if not short:

CM.append([-p for $\mathrm{p}$ in ZeroRow $])$

if full:

return matrix $(\mathrm{CM})$

else:

return matrix $(\mathrm{CM})[:, 1:]$

This was used principally to test whether constraint matrices were unimodular or totally unimodular. 


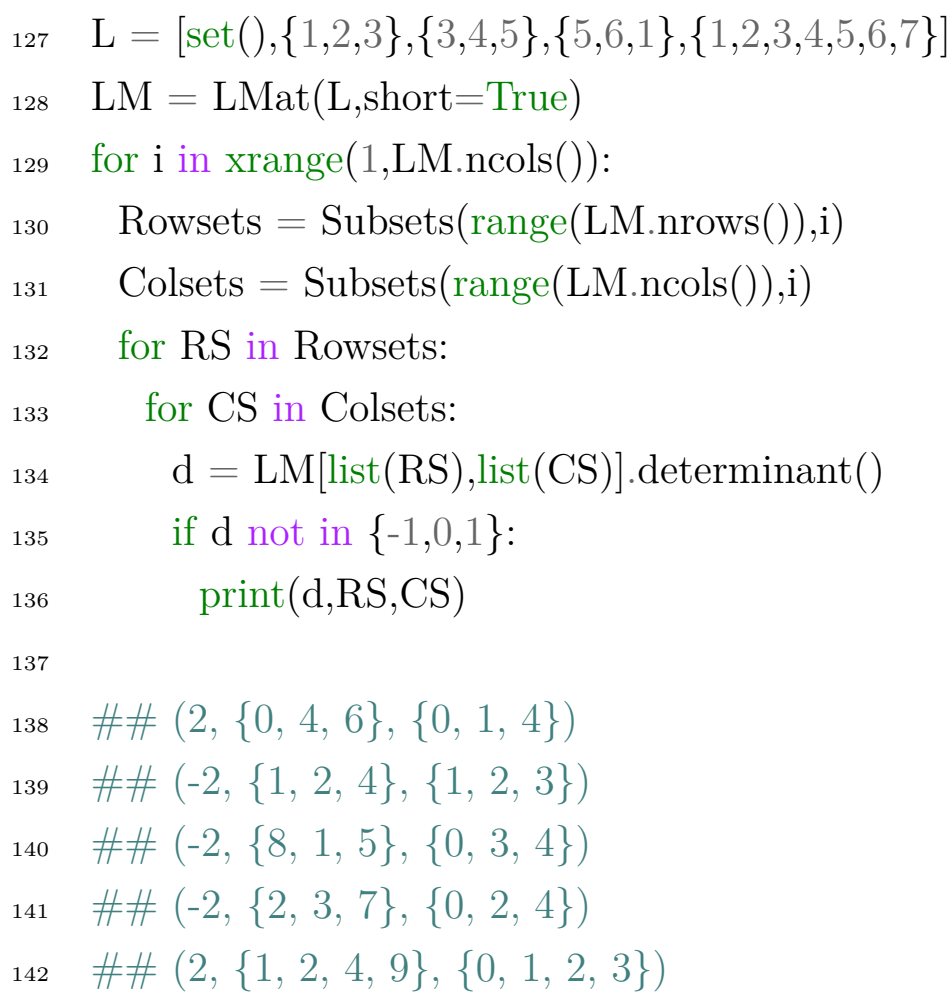

The CyclicFlats function finds the lattice of cyclic flats of an input matroid, based on the fact that a flat is cyclic if and only if its complement is a coflat. This was used to find known matroidal lattices of sets.

def CyclicFlats(M):

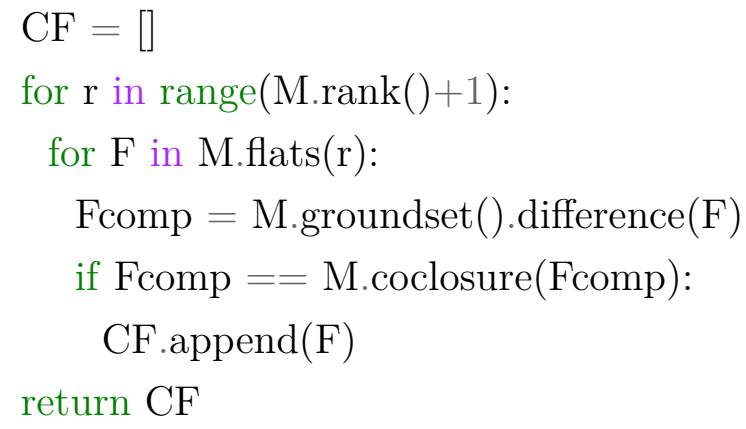

The CFMatroid function generates a matroid when given a collection of cyclic flats and their ranks. It uses the largest set in the collection as the ground set of the matroid. It generates a matroid based on a characterisation 
of matroid circuits in terms of cyclic flats and their ranks: if $M$ is a matroid on $E$, then $C \subseteq E$ is a circuit of $M$ if and only if $C$ is minimal in $E$ with regard to the property that there is a cyclic flat $Z$ of $M$ where $C \subseteq Z$ and $|C|=r(Z)+1[1]$.

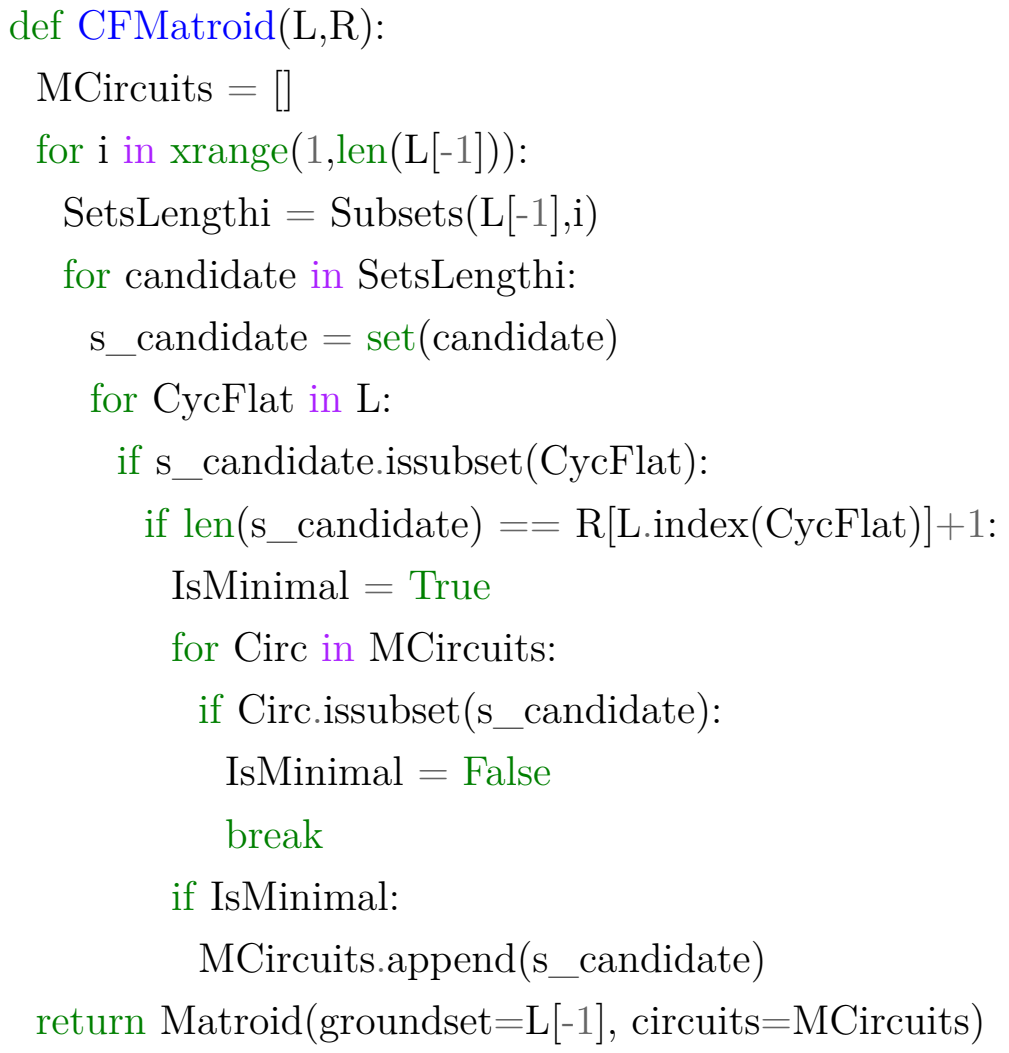

The LatticeProgram function generated a linear program object from a lattice given as input.

def LatticeProgram(L):

Lat $=$ true

$\mathrm{f}=\operatorname{len}(\mathrm{L})$

Prog $=$ MixedIntegerLinearProgram $($ maximization $=$ True $)$

Var=Prog.new_variable(nonnegative=True,name="z")

\#Add the constraint that the zero of the lattice is assigned zero.

Prog.add_constraint $(\operatorname{Var}[0]==0)$ 


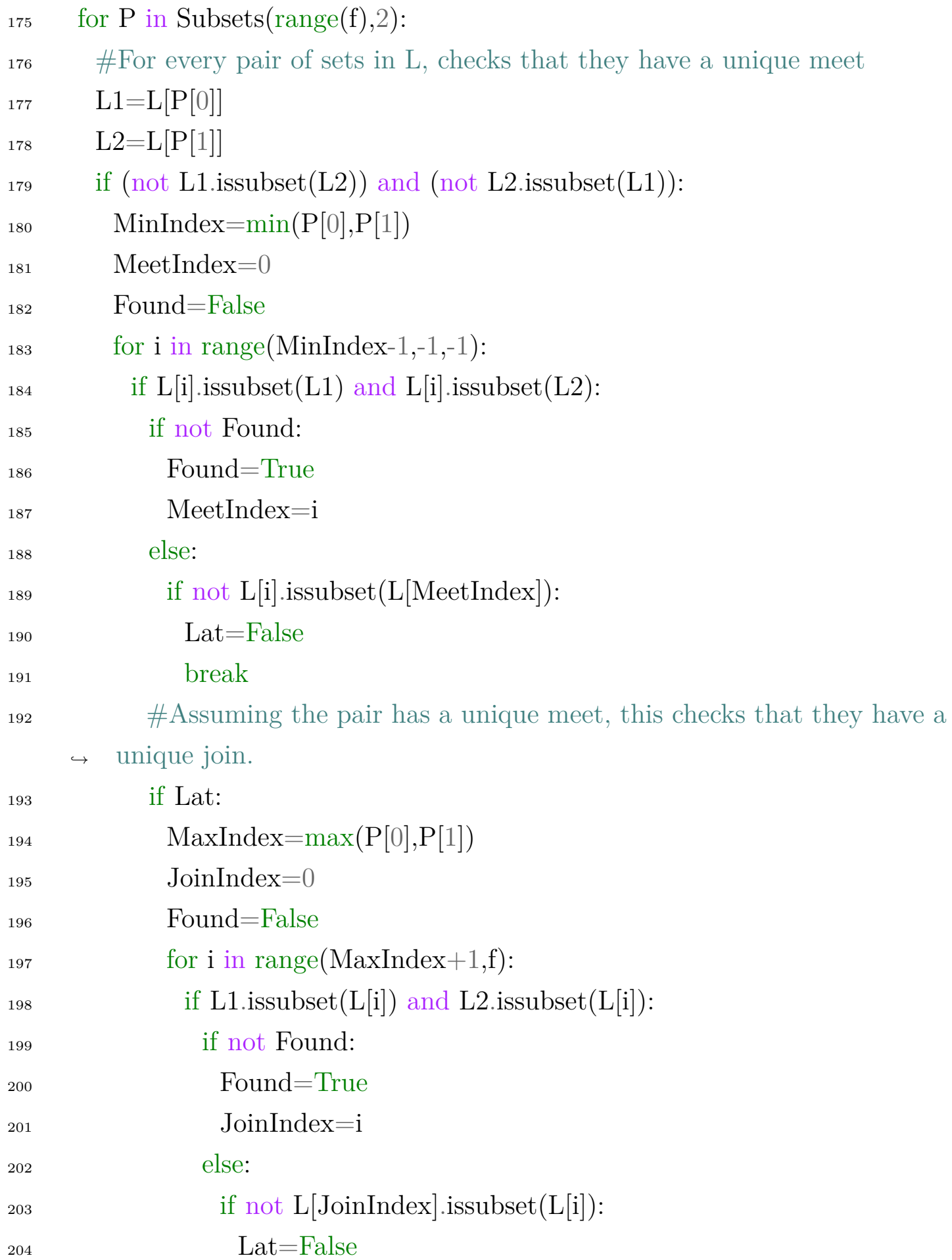




\section{APPENDIX A. SOFTWARE}

break

\#Assuming that they have a unique meet and join, add the

$\hookrightarrow$ submodular constraint for this pair to the program.

if Lat:

Prog.add_constraint $(\operatorname{Var}[\mathrm{P}[0]]+\operatorname{Var}[\mathrm{P}[1]]>=\operatorname{Var}[$ JoinIndex $]+$

$\hookrightarrow \operatorname{Var}[$ MeetIndex] + len(L1.intersection(L2)) - len(L[MeetIndex])) else:

break

\#Assuming that $\mathrm{L}$ is a lattice, this adds the two constraints arising from

$\rightarrow$ each covering relation in the lattice.

if Lat:

for $\mathrm{i}$ in range(f):

for $\mathrm{j}$ in range(f):

$\mathrm{Li}=\mathrm{L}[\mathrm{i}]$

$\mathrm{Lj}=\mathrm{L}[\mathrm{j}]$

if $\operatorname{Cover}(\mathrm{Li}, \mathrm{Lj}, \mathrm{L})$ :

Prog.add_constraint(Var[i]-Var[j] $>=1)$

Prog.add_constraint(Var[i]-Var[j] $<=\operatorname{len}(\mathrm{Li})-\operatorname{len}(\mathrm{Lj})-1)$

Prog.set_objective(Var[0])

return Prog

else:

return False

The following code was used to generate random collections of subsets, test if they were lattices, and generate the vertices of their polyhedra if they were. It allowed examination of a great many lattices to test their properties.

$\mathrm{n}=24$

$\mathrm{m}=8$

$\mathrm{p}=0.5$

$\mathrm{c}=5$

$\mathrm{E}=\operatorname{set}(\operatorname{range}(\mathrm{n}))$ 


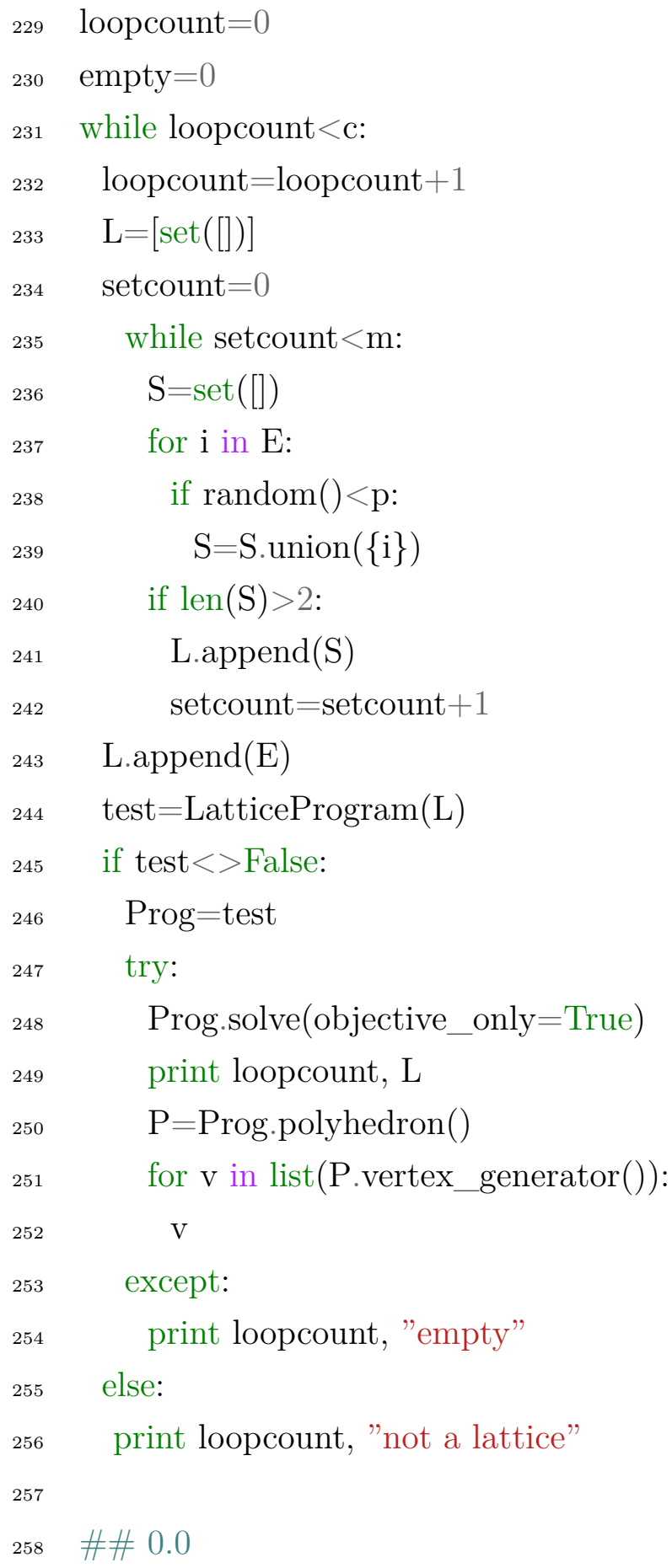




\section{APPENDIX A. SOFTWARE}

259

260

261

262

263

264

265

266

267

268

269

270

272

273

274

275

276

277

\#\# 1 [set([]), set([1, 2, 3, 4, 8, 9, 10, 12, 13, 16, 17, 18, 21, 22]), set([0, 2, 3, $\hookrightarrow \quad 5,7,8,10,14,17,19,20,22,23])$, set([1, 4, 5, 9, 10, 12, 15, 17, 18, 20, $\hookrightarrow \quad 21,22,23]), \operatorname{set}([0,1,3,5,6,7,9,10,13,14,15,16,17,18,19,21$,

$\hookrightarrow \quad 23]), \operatorname{set}([0,2,3,4,6,9,12,13,14,16,17,18,19,20,21,22]), \operatorname{set}([1,3$, $\hookrightarrow 4,7,8,9,11,12,14,16,17,22])$, set $([0,1,3,4,6,9,10,11,12,13,14$, $\hookrightarrow 18,19,21,23]), \operatorname{set}([0,1,2,4,5,7,8,12,19]), \operatorname{set}([0,1,2,3,4,5,6,7$, $\hookrightarrow \quad 8,9,10,11,12,13,14,15,16,17,18,19,20,21,22,23])]$

\#\# A vertex at $(0,12,12,14,11,13,13,11,13,8)$

\#\# A vertex at $(0,13,12,14,10,13,12,11,13,8)$

\#\# A vertex at $(0,13,12,14,10,13,13,11,13,8)$

\#\# A vertex at $(0,13,12,14,12,13,12,11,13,8)$

\#\# A vertex at $(0,13,12,14,12,13,13,11,13,8)$

\#\# A vertex at $(0,12,12,14,12,13,13,11,13,8)$

\#\# 2 empty

\#\# 3 empty

\#\# 4 empty

\#\# 0.0

\#\# $5[\operatorname{set}([]), \operatorname{set}([0,2,3,5,6,7,8,13,14,15,17,18,22,23]), \operatorname{set}([2,4,7$,

$\hookrightarrow 9,11,12,13,14,15,16,20,21,23])$, set $([16,1,3,17,8,23,13,14])$,

$\hookrightarrow \operatorname{set}([1,2,6,7,9,10,12,14,16,17,18,19,20,23]), \operatorname{set}([0,1,2,3,6,8$,

$\rightarrow 9,14,15,16,21,22,23])$, set $([3,4,5,6,7,10,11,12,13,15,16,17$,

$\hookrightarrow 18,20]), \operatorname{set}([4,5,22,7,9,13,14,15]), \operatorname{set}([2,4,6,10,11,17,18,19$,

$\hookrightarrow \quad 20,22]), \operatorname{set}([0,1,2,3,4,5,6,7,8,9,10,11,12,13,14,15,16,17,18$,

$\rightarrow \quad 19,20,21,22,23])]$

\#\# A vertex at $(0,10,10,11,7,10,10,9,7,9)$

\#\# A vertex at $(0,10,10,11,7,9,10,10,7,9)$

\#\# A vertex at $(0,11,11,12,7,11,11,10,7,9)$

\#\# A vertex at $(0,11,11,12,7,10,11,11,7,9)$

\#\# A vertex at $(0,11,11,12,7,10,11,10,7,9)$

\#\# A vertex at $(0,11,11,12,7,11,11,11,7,8)$

\#\# A vertex at $(0,10,10,11,7,10,10,10,7,8)$ 
278 \#\# A vertex at $(0,10,10,11,7,19 / 2,10,19 / 2,7,17 / 2)$

$279 \quad \# \#$ A vertex at $(0,10,10,11,7,10,10,10,7,9)$

280 \#\# A vertex at $(0,11,11,12,7,11,11,11,7,9)$ 


\section{Bibliography}

[1] Bonin, J. E., and de Mier, A. The lattice of cyclic flats of a matroid. Annals of Combinatorics 12, 2 (July 2008), 155-170.

[2] Crapo, H., and Schmitt, W. The free product of matroids. European Journal of Combinatorics 26, 7 (October 2005), 1060-1065.

[3] Davey, B. A., and Priestley, H. A. Introduction to Lattices and Order, 2nd ed. Cambridge University Press, 2002.

[4] Karp, R. M. Reducibility among Combinatorial Problems. Springer, 1972, ch. 9, pp. 85-103.

[5] Schrijver, A. Theory of linear and integer programming. WileyInterscience Series in Discrete Mathematics and Optimization. John Wiley \& Sons Ltd, 1998.

[6] Sims, J. A. Some Problems in Matroid Theory. PhD thesis, Linacre College, Oxford University, 1980. 